NBER WORKING PAPER SERIES

\title{
HISTORY AS EVOLUTION
}

\author{
Nathan Nunn \\ Working Paper 27706 \\ http://www.nber.org/papers/w27706
}

\author{
NATIONAL BUREAU OF ECONOMIC RESEARCH \\ 1050 Massachusetts Avenue \\ Cambridge, MA 02138 \\ August 2020
}

I am particularly grateful to Joseph Henrich for numerous insightful discussions. I also thank Alberto Bisin, Robert Boyd, Ben Enke, Michele Gelfand, Michael Muthukrishna, James Robinson, and Felipe Valencia Caicedo for helpful comments and/or discussions. I also thank Vafa Behnam, Aditi Chitkara, Rumi Khan, and Laura Kincaide for excellent RA work. The views expressed herein are those of the author and do not necessarily reflect the views of the National Bureau of Economic Research.

NBER working papers are circulated for discussion and comment purposes. They have not been peer-reviewed or been subject to the review by the NBER Board of Directors that accompanies official NBER publications.

(C) 2020 by Nathan Nunn. All rights reserved. Short sections of text, not to exceed two paragraphs, may be quoted without explicit permission provided that full credit, including () notice, is given to the source. 
History as Evolution

Nathan Nunn

NBER Working Paper No. 27706

August 2020

JEL No. C73,N01,N10,Z1

\begin{abstract}
$\underline{\text { ABSTRACT }}$
In this chapter, I consider the benefits of viewing history through an evolutionary lens. In recent decades, a field of research has emerged, which builds on foundations from biological evolution to study culture within an evolutionary framework. I begin the chapter by discussing the theory behind cultural evolution and the empirical evidence supporting its ability to explain the history of human societies. I then turn to a discussion of how an evolutionary perspective provides important insights into a range of phenomena within economics, including a deeper understanding of human capital, innovation, gender roles, the consequences of warfare, the effects of market competition, why we observe historical persistence and path dependence, and, most importantly, why sustained economic growth is often so elusive. I end by turning to a summary of a growing body of research within economics that has made progress in improving our understanding of cultural evolution and, thus, contributing to evolutionary disciplines outside of economics.
\end{abstract}

Nathan Nunn

Department of Economics

Harvard University

1805 Cambridge $\mathrm{St}$

Cambridge, MA 02138

and NBER

nnunn@fas.harvard.edu 


\section{Contents}

1 Introduction

2 The Benefits of Cultural Evolution $\quad 5$

A Cultural evolution saves on information costs $\ldots \ldots \ldots \ldots$

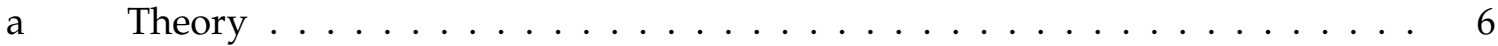

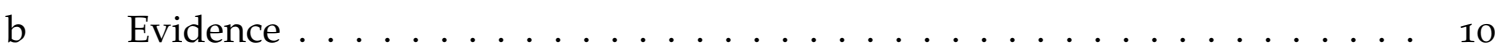

B Cultural evolution is cumulative $\ldots \ldots \ldots \ldots \ldots \ldots \ldots$

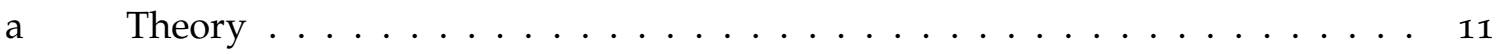

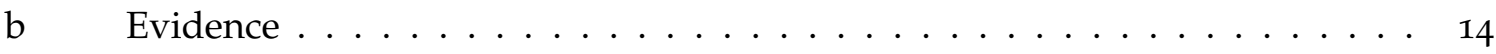

3 Insights from a Recognition of History as Evolution $\quad 16$

A Environmental Mismatch $\ldots \ldots \ldots \ldots \ldots \ldots \ldots$

a $\quad$ Endogenous Mismatch . . . . . . . . . . . . . . . 22

B Education and Cultural Evolution $\ldots \ldots \ldots \ldots \ldots \ldots$

a Innovation and the Collective Brain $\ldots \ldots \ldots \ldots$

C How and Why History Matters . . . . . . . . . . . . . . 34

a Kludges ............................ 40

D Group-Level Selection . . . . . . . . . . . . . . . . . . 44

E Biology, Sex, and Gender . . . . . . . . . . . . . . . . 49

4 Contributions of Economics to Cultural Evolution $\quad 51$

5 Conclusions $r$

$\begin{array}{lr}\text { References } & 58\end{array}$ 


\section{List of Figures}

$1 \quad$ The equilibrium proportion of traditionalists $(\mathrm{T})$ and non-traditionalists (NT). . . 9

2 The adoption of a new (optimal) action following a shock depends on the proportion traditionalists in the society $x^{*} \ldots \ldots \ldots \ldots \ldots \ldots$

3 An example of environmental mismatch: The dodo bird (raphus cucullatus). Sketch by Roelandt Savery, 1626. Source: Figure 10 of Hume (2006) . . . . . . . . . . . . . 19

4 The equilibrium proportion of traditionalists (T) and non-traditionalists (NT), assuming the instability of the environment is endogenous to learning by NT (and

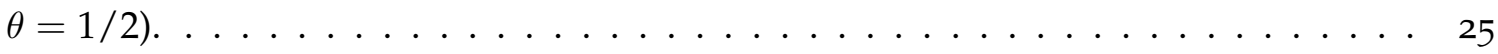

5 Illustration of the model of cumulative culture / human capital developed by Henrich $(2004 b) \ldots \ldots \ldots \ldots \ldots$

6 Example of a mountain landscape dictating the evolution of the eye. Source: Figure

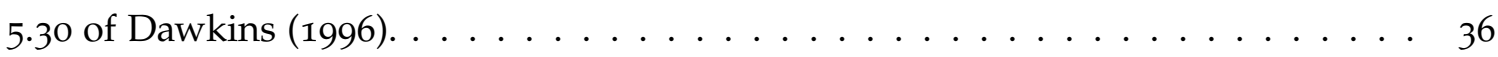

7 Temporary historical events and permanent effects. . . . . . . . . . . . 38

8 An example of a kludge: the bony flatfish. Source: Figure 1 of Norman (1934). . . . 41

\section{List of Tables}

I Summary of the literature on the long-term determinants of cultural traits. . . . . 53

2 Overview of the economics literature on the shorter-term determinants of cultural

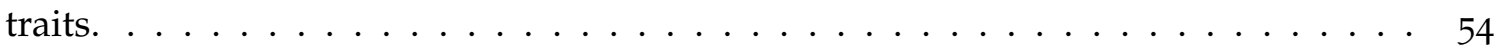

3 Overview of the economics literature on the consequences of differences in cultural

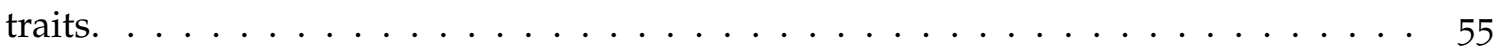




\section{Introduction}

To the typical economic historian, the relevance of evolutionary perspectives, such as those from evolutionary biology, evolutionary psychology, or evolutionary anthropology, to the study of economic history of long-term economic growth likely seem limited. The importance of evolution or biology may seem restricted to well-defined subfields within economics that study the importance of genetics for economic outcomes. ${ }^{1}$ However, in this chapter, I will argue that an evolutionary perspective can provide further and more widely-relevant insights for the study of economic history and long-run economic growth. In particular, my aim is to reduce the perceived gap between research done within the field of economic history and that done within the fields of evolutionary biology, evolutionary psychology, and evolutionary anthropology.

The field of evolutionary research that is the most relevant for economic history is cultural evolution. The field was an outgrowth of evolutionary biology and emerged as a line of research interested in better understanding human psychology, human societies, human behavior, and their evolution over time. The first contributions were theoretical studies, such as Cavalli-Sforza and Feldman (1981) and Boyd and Richerson (1985), that adapted and extended models from evolutionary biology and applied them to cultural evolution. ${ }^{2}$

I begin the chapter by first providing a conceptual and theoretical description of culture and cultural evolution. An important part of this is to describe the theory and evidence behind the benefits of culture and why it is such a central part of human decision making and, therefore, the fabric of human society. As I will discuss, there are two aspects of culture and its evolution that are particularly important. The first is that culture is efficient. By relying on traditions that are passed down over generations, individuals are able to effortlessly make decisions in complex environments where figuring out the optimal action with certainty is costly or even impossible. When a population's setting is stable over time, then reliance on evolved cultural traditions is an effective strategy.

\footnotetext{
${ }^{1}$ Examples of this line of research includes geneoeconomics (Benjamin, Cesarini, Chabris, Glaeser, Laibson, Guonason, Harris, Launer, Purcell, Smith, Johannesson, Magnusson, Beauchamp, Christakis, Atwood, Hebert, Freese, Hauser, Hauser, Grankvista, Hultman and Lichtenstein, 2012), macro-level analyses of the effects of genetic distance and genetic diversity (Spolaore and Wacziarg, 2009, Ashraf and Galor, 2012), micro-level studies on the importance of genetics relative to the environment for economic outcomes (Sacerdote, 2007, Cesarini, Dawes, Fowler, Johannesson, Lichtenstein and Wallace, 2008, 2009), or empirical and theoretical research that takes a Darwinian perspective to understand economic growth (Galor and Moav, 2002, Clark, 2007).

${ }^{2}$ For an overview and introduction into the field of cultural evolution, see Chudek, Muthukrishna and Henrich (2015). For a description of the history of the field, see Mesoudi (2016).
} 
The second aspect of cultural evolution is that it is cumulative. Culture allows societies to accumulate an evolved body of knowledge that is greater than any single individual could learn within their lifetime or fit within their mind. By taking as given the cultural wisdom of previous generations, societies do not need to 'reinvent the wheel' and instead can focus their efforts on adding to the body of culturally accumulated knowledge of the society - i.e., what has been called the 'collective brain' (Muthukrishna and Henrich, 2016).

Having described the conceptual foundations of cultural evolution and its benefits, I then turn to a series of examples aimed at showing how an evolutionary perspective of human behavior can provide insight into the study of economics in general and economic history in particular. I do this in three ways. The first is to show that the differences between evolutionary thinking and traditional economic thinking are often much smaller than one might think. Specifically, there are many cases where the same logic, the same evidence, and a similar narrative are developed but using different terminology, empirical methods, and data. Thus, I hope to make these commonalities more apparent to the reader.

The second strategy is to highlight instances in which the insights from the evolutionary literature can be leveraged by economic historians to gain a better understanding of historical processes. Here, I will touch on a few cases that have become apparent to me over the years. I will discuss how the evolutionary insights, such as environmental mismatch, the collective brain, cumulative improvements, kludges, group-level selection, sexual dimorphism, and reproduction strategies, helps us develop a deeper understanding of a diverse set of aspects of human history, including human capital, innovation, warfare, state formation, cooperation, social structure, gender roles, kinship, social structure, path dependence, and comparative economic development. To me, these are the most obvious examples of the insights that emerge from an evolutionary perspective. However, my sense is that they are just the tip of the iceberg.

The third strategy is to highlight cases in which historical research within economics has contributed to the cultural evolution literature. As I will discuss, a sizeable and quickly growing area of research within economics has taken an evolutionary perspective, thus providing important contributions, both theoretical and empirical, to evolutionary fields outside of economics. 


\section{The Benefits of Cultural Evolution}

Given my argument about the importance of cultural evolution for studying the history of human societies, it is necessary to first define what is meant by the term 'culture'. The standard definition from evolutionary anthropology defines culture as the knowledge, technology, values, beliefs, and norms that can be transmitted across generations and between individuals (e.g., Boyd and Richerson, 1985). There are numerous examples of cultural that vary by context, but examples include religious/supernatural beliefs, views about morality, norms about giving and cooperation, gender norms, food preferences, taboos, and traditions and skills regarding farming, house-building, hunting, etc.

Implicit in this definition is that our culture influences the decisions we make and therefore affects human behavior. For scholars in evolutionary fields, this is natural and an obvious fact. However, for economists this is not something that we take for granted. Instead, when one views human behavior from the traditional economic perspective of 'rationality', particularly of one takes a narrow definition of rationality, then a natural question arises: why does culture exist? Why would someone be influenced by what they are told by their teachers, their parents, friends, church leaders, celebrities, etc? Why wouldn't individuals just figure out what is best on their own, engaging in a form of rational calculus? For example, if cheating, stealing, or lying yields a higher payoff, then why would someone be influenced by the fact that religious leaders, parents, teachers, or friends might tell them that this behavior is wrong and should be avoided? These are important questions and to understand the logic behind culture and its benefits, we now turn to a theoretical examination of culture. Specifically, I now discuss a theoretical literature where culture is not taken as given, but is derived endogenously. In other words, an important characteristic of the models is that they show when individuals would use culture to guide their decision and why doing this can be beneficial.

\section{A. Cultural evolution saves on information costs}

To understand the primary benefits of culture, one must first recognize an important fact: As human beings, we have cognitive limits. Acquiring and processing information has an opportunity cost. In the face of these limits, we have developed culture and cultural learning, and with them cultural values and beliefs. These serve as tools that are "fast and frugal" and allow us to make 
decisions more efficiently than if we were an economist's traditional version of 'rationality' (e.g., Gigerenzer and Goldstein, 1996, Todd and Group, 1999).

\section{a. Theory}

The formal theory behind culture and its evolution has been well developed in the cultural evolution literature and began with seminal models by Boyd and Richerson (1985) and Rogers (1988) among others. ${ }^{3}$ The authors model situations where an action must be made in a setting without perfect (costless) certainty. The payoff of each action depends on the environment, which is variable. Individuals can either collect information and figure out the optimal action on their own or they can rely on the traditions that have evolved up until the previous generation. They do this by choosing the action of a person from the previous generation. This effectively models the process of transmission of cultural or psychological traits across generations. Under very general conditions, there will always be some proportion of the population that relies on the cultural traditions of the previous generation.

The models show that, under fairly general conditions, we should observe the presence of culture and decision-making that are based on cultural values. There are two primary benefits that culture provides over rationality. First, culture-based decision-making provides a quick and easy way to make decisions. To the extent that rational decision-making (narrowly defined) requires costs due to information acquisition or cognitive processing, then acting on one's transmitted cultural traditions and values saves on these costs. The second benefit is that relying on culture allows for cumulative learning. By following the culture of the previous generations, individuals do not have to reinvent the wheel and re-learn everything that has already been figured out during the history of the society in question. For example, if the society has already learned how to effectively hunt, which plants are not poisonous, and what rituals and beliefs helps the society to exist in harmony, then taking these as given and trying to improve upon them may be a better strategy than having individuals try to figure these things out again.

I now present a simple model which focuses on the first of the two benefits and shows how culture can emerge in equilibrium. Following this, I discuss the second benefit of culture, the fact that it is cumulative, in Section $2 \mathrm{~B}$.

\footnotetext{
35or a related paper within economics, see Bisin and Verdier (2001a). The models are very similar, except that what we think of as a reliance on culture/tradition in the description below they interpret (and model) as imperfect empathy on the part of the parents.
} 
The model is taken from Giuliano and Nunn (forthcoming) and reproduces the basic logic of Rogers (1988), which is one of the first and simplest models of this aspect of cultural evolution. The players of the game consist of a continuum of members of a society. Each period, a new generation is born and the previous generation dies. When a player is born, they make a onceand-for-all choice of two possible actions, which we denote $a$ and $b$. Which of the two actions yields a higher payoff depends on the state of the world (i.e., the environment), which can be either $A$ or $B$. If the state is $A$, then action $a$ yields the payoff $\beta>0$ and action $b$ yields a payoff of $-\beta$. If the state is $B$, then action $a$ yields a payoff of $-\beta$ and action $b$ yields the payoff $\beta>0$. Thus, in each state, one of the two actions is better than the other.

In each period, with probability $\Delta \in[0,1]$, there is a shock which results in a new draw of the state of the environment. It is equally likely that the draw results in the new environment being in state $A$ or state $B$. The state of the world is unknown to the players. However, as I explain below, it is possible to engage in learning (at a cost) to determine the state of the world.

There are two potential types of players. Each uses a different method to choose their action. The first type, "Traditionalists (T)," value tradition and place strong importance on the actions of the previous generation. They choose their action by following the action of a randomly chosen person from the previous generation. Thus, the model allows for both vertical and oblique transmission. 4 The second type, "Non-Traditionalists (NT)," do not value tradition and ignore the actions of the previous generation. They obtain the optimal action with certainty for the current period, but there is a cost of learning, $\kappa \in(0, \beta)$. Thus, although the cost is positive it is assumed to be fairly modest. Let $x$ denote the proportion of the population that is traditionalists.

I now turn to an examination of the payoffs of both types of players, starting with the nontraditionalists. In each generation, they incur the cost $\kappa$ to learn the optimal action. This action is chosen and they obtain $\beta$. Therefore, the payoff to a non-traditionalist is given by:

$$
\Pi^{N T}=\beta-\kappa
$$

To calculate the expected payoff of a traditionalist, consider the following sequence of possibilities, each of which results in a traditionalist choosing the right action for her environment, thus, receiving $\beta$. First, a traditionalist copies a non-traditionalist from the previous generation; and the environment did not experience a shock between the last and current generation. Since

\footnotetext{
4Vertical transmission is transmission from parents to children. Oblique transmission is all other forms of transmission of those from an older generation to the younger generation.
} 
the non-traditionalist from the previous generation chose the action that was optimal in her environment and since a shock did not occur, then this action will also be optimal in the current environment and the traditionalist receives $\beta$. This scenario occurs with probability $(1-x)(1-\Delta)$. Second, a traditionalist copies a traditionalist from the previous generation, who had copied a non-traditionalist from the previous generation. No shocks occurred during this time. The traditionalist receives $\beta$ and this occurs with probability $x(1-x)(1-\Delta)^{2}$. Third, a traditionalist copies a traditionalist, who copied a traditionalist, who copied a non-traditionalist. No shocks occurred during this time. This occurs with probability $x^{2}(1-x)(1-\Delta)^{3}$.

One can continue this sequence of possibilities infinitely. The sum of the probabilities is given by $\sum_{t=1}^{\infty} x^{t-1}(1-x)(1-\Delta)^{t}$. With probability equal to one minus this sum, a traditionalist does not necessarily obtain the correct action. In these cases, there has been at least one shock to the environment since the most recent non-traditionalist was copied. Because the consequence of a shock is an equal probability of being in either state, a traditionalist still has a $50 \%$ chance of receiving $\beta$, a $50 \%$ chance of receiving $-\beta$, and her expected payoff is 0 . Putting this together, and using the formula for an infinite geometric sequence gives:

$$
\Pi^{T}=\frac{\beta(1-x)(1-\Delta)}{1-x(1-\Delta)} .
$$

The payoffs to traditionalists and non-traditionalists as a function of the proportion of traditionalists in the society, $x$, are shown in Figure 1 . The expected payoff of a traditionalist, $\Pi^{T}$, is decreasing in $x$. Intuitively, as the fraction of traditionalists increases, it is less likely that a traditionalist will copy a non-traditionalist who is more likely to have chosen the correct action. At the extreme, where everyone in the population is a traditionalist $(x=1)$, each traditionalist copies another traditionalist and the expected payoff is 0 .

At the other extreme, where everyone is a non-traditionalist $(x=0)$, a (mutant) traditionalist will copy the correct action from someone in the previous generation and as long as there was not a shock to the environment between the two generations, she will obtain the right action. Thus, with probability $1-\Delta$, a traditionalist's payoff is $\beta$. If, on the other hand, the environment did change, which occurs with probability $\Delta$, then there is an equal probability that the environment is in either state and the expected payoff is 0 . Therefore, the expected payoff to a traditionalist when $x=0$ is $\beta(1-\Delta)$.

In an equilibrium with both types present, the expect payoffs to both types must be equal. In 


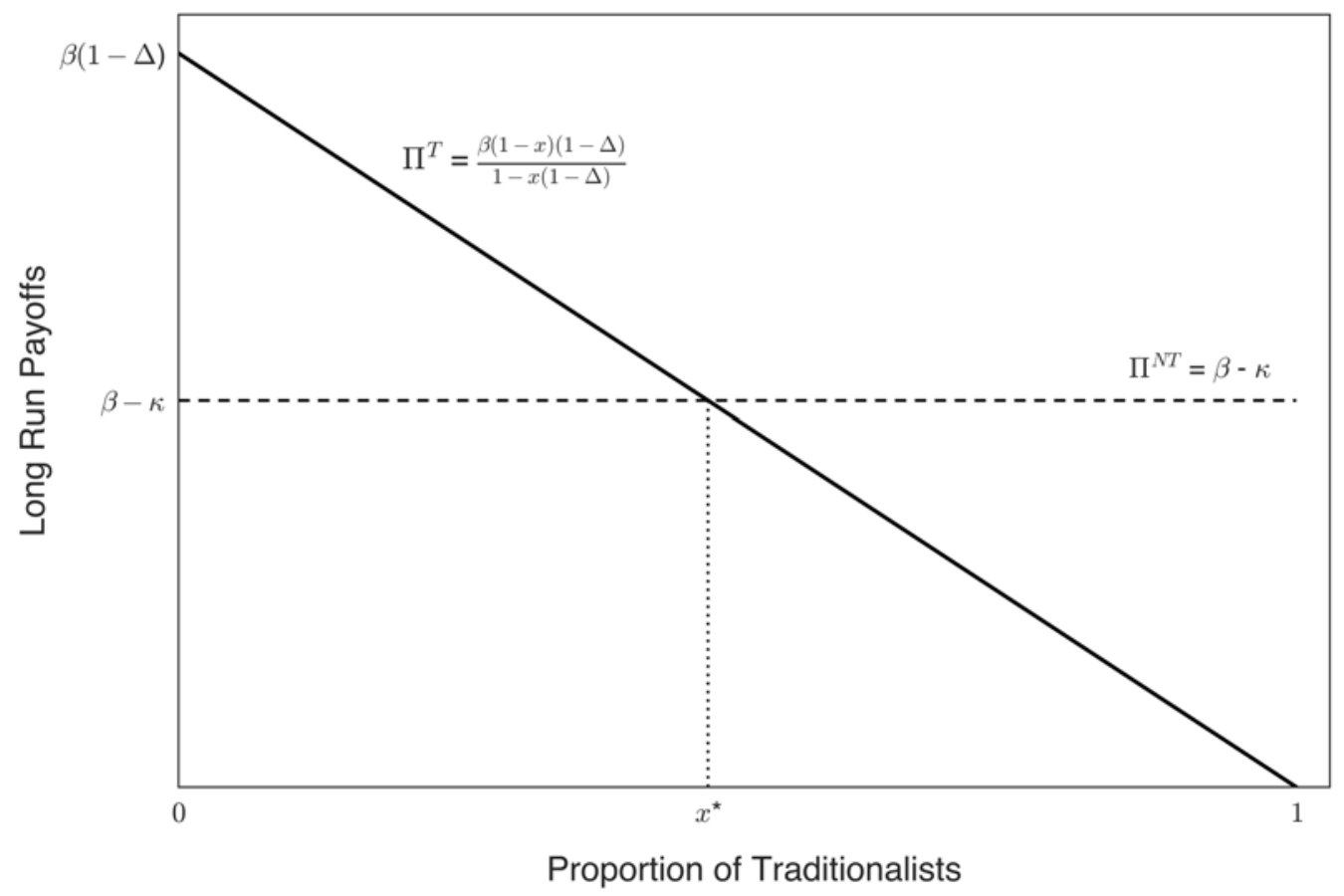

Figure 1: The equilibrium proportion of traditionalists $(\mathrm{T})$ and non-traditionalists (NT).

an equilibrium with only one type, its average payoff must be no less than that of the other type. Thus, the equilibrium proportion of traditionalists $x^{*}$ is given by:

$$
x^{*}=\left\{\begin{array}{lll}
\frac{\kappa-\Delta \beta}{\kappa(1-\Delta)} & \text { if } & \Delta \in\left[0, \frac{\kappa}{\beta}\right] \\
0 & \text { if } & \Delta \in\left[\frac{\kappa}{\beta}, 1\right]
\end{array}\right.
$$

From Figure 1, it is clear that under fairly general conditions $(\kappa>\Delta \beta)$, traditionalists are present in society. Their emergence is due to the benefit of cultural transmission, which provides a fairly accurate way of making decisions at low cost.

It is straightforward to show that the equilibrium is stable under the standard assumption that the relative payoffs of types affects their fitness and/or survival. Formally, this can be modeled using the standard replicator dynamic (e.g., Gintis, 1997). In the polymorphic equilibrium, where both types are present, a small perturbation of $x>x^{*}$, causes the payoff of traditionalists to be lower than of non-traditionalists and $x$ will decrease until $x^{*}$ is reached. If $x<x^{*}$, the payoff of traditionalists is higher than of non-traditionalists, and $x$ will increase until $x^{*}$ is reached. In the monomorphic equilibrium with $x^{*}=0$, a perturbation of $x>x^{*}$, causes the payoff of 
traditionalists to be lower than of non-traditionalists and $x$ will decrease until there is convergence to $x^{*}$.

\section{b. Evidence}

In the model, the presence of culture in equilibrium is due to the benefit of cultural transmission, which provides a fairly accurate way of making decisions at a low cost. Anthropologists have documented numerous real-world examples of functional cultural traits being followed despite the population not knowing their benefits. ${ }^{5}$ One of the best-known examples is the alkali processing of maize, which is the traditional method of preparing maize in many parts of Latin America. During the process, dried maize is boiled in a mixture of water and limestone or ash, before being mashed into a dough called 'masa'. Although it was unknown at the time, putting limestone or ash in the water before boiling prevents pellagra, a disease resulting from niacin deficiency, which occurs in diets that consist primarily of maize. The alkaline solution that is created when limestone or ash is added increases the body's absorption of niacin (Katz, Hediger and Valleroy, 1974).

Another example of the benefits of culture and tradition is documented in Billing and Sherman (1998). The authors examine data for 43 spices from 4,578 meat-based recipes in 93 cookbooks from 36 countries. They document several empirical regularities that are consistent with the effective use of spices as antimicrobials even though this benefit was unknown. They show that the spices that are more commonly used are the ones that are more antimicrobial. Societies in hotter climates use more antimicrobial spices. Spices are used in ways, and in combinations, that appear to maximize their antimicrobial properties. For example, onions are not effective unless they are cooked and cilantro is not effective if it is cooked. In most recipes, onions are cooked and cilantro is not. Another example is chili powder (e.g., red pepper, onion, garlic, cumin, etc) which contains a combination of the spices that generate complementarities and maximizes their effectiveness.

Another well-known example of a tradition with functional benefits that were unknown is from the Naskapi, who are an indigenous First Nations society who traditionally lived on land that today is in Quebec, New Foundland and Labrador (Speck, 1935). The primary form of subsistence of the Naskapi was caribou hunting. Deciding where to hunt is an important decision. The

${ }^{5}$ For an excellent overview and many examples, see chapter 7 of Henrich (2016). 
hunters would like to hunt in the locations where the caribou are. By contrast, the caribou would like to avoid the locations where the hunters are. Effectively, this is a two-dimension version of the game "matching pennies."6 We know that in such a game, the only Nash equilibrium is a mixed strategy equilibrium where one randomly chooses each direction with equal probability. The difficulty is that human beings are notoriously bad at randomizing and instead would tend to follow certain patterns, which could be detected by the Caribou. The Naskapi developed a ritual that they undertook prior to hunting expeditions. They would put the shoulder blade of a caribou in the fire. It would then burn and crack and the patterns of these told the hunters where they should hunt. Although this was unknown, the ritual was effective because it provided a method to randomly select the location of the next hunting expedition.

The logic of the models has been tested and confirmed in numerous studies. One clear prediction of the models is that in equilibrium, there should be a strong reliance on culture and tradition (i.e., more social learning) in environments that are unstable and for which determining the optimal action is difficult. Within the evolutionary literature, this prediction has been tested using experimental tools (Galef and Whiskin, 2004, McElreath, Lubell, Richerson, Waring, Baum, Edstein, Efferson and Paciotti, 2005, Toelch, van Delft, Bruce, Donders, Meeus and Reader, 2009).

Most recently, the prediction that tradition and cultural persistence should be weaker in more unstable environments was tested by Giuliano and Nunn (forthcoming). The study uses paleoclimatic data, combined with information on the historical locations of ethnic groups, to construct estimates of the variability of the ancestral environment across generations for ethnic groups and countries. They find that ancestral climatic stability is associated with greater self-reported importance placed on tradition, and more persistence in cultural traits over time, including among the descendants of immigrants to the United State and Indigenous populations from the United States and Canada.

\section{B. Cultural evolution is cumulative}

\section{a. Theory}

An important characteristic of the model discussed above, which has long been recognized in the literature, is that, in the end, the existence of culture and tradition does not make the society

\footnotetext{
${ }^{6}$ Matching pennies is a two-player zero-sum game in which each player chooses either heads or tails. One player obtains a higher payoff when the players' choices are the same. The other player obtains a higher payoffs the the players' choices are different.
} 
better off. In the long-run, regardless of the extent to which culture is present, the society-wide payoff is $\beta-\kappa$. This is contrary to the conventional wisdom that humans are more successful than other animals because we have culture, which leads to greater group-level success (Henrich, 2016). Subsequent theoretical work has shown that this characteristic of Rogers' model is not due to its simplicity. Instead, it is general and found in a large class of models where the only benefit of culture is to save on individual-level information acquisition (Boyd and Richerson, 1995, 2005).

This has led to an emphasis on 'cumulative cultural evolution' as a key benefit of culture. It is related to the benefit of information acquisition, highlighted in the model above but conceptually distinct. To put it simply (and to use multiple clichés), an important benefit of culture it that it means that we do not have to 'reinvent the wheel' and that we can 'stand on the shoulders of giants.' We can take as given the knowledge or traditions of the previous generation, without necessarily understanding them fully, and build on them, continuing the process of incremental cultural innovation.

To see the logic of this, consider the following variant of the model above, which is detailed in Boyd and Richerson $(1985,1995)$. The set up is the same except there is a continuum of states. As before, in each period, there is a probability that the environment switches to a new state. There is also a continuum of behaviors, with one behavior yielding the highest payoff for each possible state. In addition, the payoff of a behavior in a particular state is decreasing in the distance from the state's optimal behavior. We will see examples below, but concretely one can think of the behavior as being the technology used to build tools or houses, or strategies used to forage for food or hunt for game. The further one deviates from the optimal strategy or technology, the lower is one's expected payoff.

Unlike the model above, it is now assumed that all individuals can modify their behavior by learning. Individuals start with an initial guess and then through costly trial and error modify their behavior. Following the same logic as above, there are two types: traditionalists and non-traditionalists. Traditionalists adopt the behavior of a randomly chosen individual from the previous generation and use this as the starting point from which they experiment. By contrast, non-traditionalists ignore the behavior from the previous generation and use a fixed behavior as their starting point and they always acquire the optimal behavior given the current state. Relative to non-traditionalists, traditionalists invest much less in changing their behavior and thus they improve upon their initial behavior much less than a non-traditionalist. 
The logic of the equilibrium of this model is similar to the prior model. As long as the environment doesn't change too frequently, traditionalists will slowly converge to the optimal behavior over time. The movement is not as rapid as for non-traditionalists, who accomplish this in one generation, but learning costs are reduced. In this model, each new generation of nontraditionalists 'reinvents the wheel' and obtains the optimal action. By contrasts, traditionalists build on the knowledge accumulated by the previous generation. Their behavior does not track the environment as optimally as non-traditionalists, but they save on information acquisition costs. In this model, the average payoff in society is increasing in the share of traditionalists in the population. Thus, the model is consistent with cultural evolution increasing the effective knowledge and wellbeing of the society.

The benefits of cumulative cultural evolution become even more clear when one recognizes that the world is much more complicated than the stylized models that we use in economics. We typically model settings where the number of determinants is modest, payoffs functions are smooth and continuous, and therefore, equilibria are typically unique and nicely behaved.7 However, reality is much more complicated. There are often many equilibria. Our wellbeing is affecting by a very large number of determinants of determinants, including our own actions, the actions of others, exogenous shocks. In addition, there are complicated interactions between each of these factors. Unlike our simplified models that feature smooth and well behaved payoff functions that generate an optimal action that is easily calculated, in reality, payoff functions are not smooth and are highly irregular. (We will see examples of this below.) In such a setting, calculating the optimal action is literally impossible. Cumulative cultural evolution reduces these much larger optimization problems into smaller, more manageable chunks, where cumulative learning can take place. This allows each generation to 'tinker' and develop small piecemeal improvements to the current values, beliefs, or technologies (i.e., culture) of a society. In addition, these simpler chunks feature simpler problems and smoother surfaces. As Boyd, Richerson and Henrich (2013, p. 136) put it:

In a small neighborhood in design space, the performance surface is approximately flat, so that even if small changes are made at random, half of them will increase the payoff (unless the design is already at the optimum). Large changes will improve

7If our models do have multiple equilibria, then we usually simplify the setting by using a selection criterion such as choosing the Pareto superior equilibrium. 
things only if they are in the small cone that includes the distant optimum. Thus, we expect it to be much harder to design a useful bow from scratch than to tinker with the dimensions of a reasonably good bow.

Thus, an important characteristic of culture is to make optimization problems that are impossible for any individual in their lifetime possible for the larger society when the problem is tackled in an incremental manner over many generations.

\section{b. Evidence}

From an evolutionary point of view, the best evidence of the importance of cumulative cultural evolution is the technological sophistication developed by humans compared to other animals including non-human primates. As early as 10,000 years ago, humans moved into and were able to subsist in diverse, and often remote, parts of the earth. Living in these environments necessitated the development of technologies like "spears, atlatls, and later bow and arrow are used to acquire game; flaked stone tools are necessary to process kills and to shape wood, bone, and process hides; clothing and shelter are crucial for thermoregulation; fire making paraphernalia is necessary for cooking, heat, and light. Slings, baskets, and pottery facilitate transport and storage; boats expand foragers; ranges to include lakes and oceans; fishhooks and cordage make coastal habitats rich sources of protein." (Boyd et al., 2013, p. 142).

Another important source of evidence on the value of cumulative cultural evolution is from numerous natural experiments where explorers (usually European) arrived in a new location with more advanced technology but no cumulative knowledge of the local environment. These "lost explorer experiments" have a remarkably similar storyline (Boyd et al., 2013, Henrich, 2016). European explorers arrive to a new location inhabited by smaller-scale societies. These are societies that the explorers perceived as being less sophisticated with technology that was much less advanced. The expedition experiences unexpected circumstances which require the explorers to remain in the new lands longer than their provisions allow for requiring them to live, as the indigenous inhabited do, off of the land. Despite having more scientific knowledge and more resources, including manufactured survival equipment, the explorers never fair well and often die. Without the benefits of cumulative cultural evolution, they are not able to survive let alone thrive in the new setting. 
One of the more notable examples among these natural experiments is the Franklin Expedition of 1846 in which the explorers starved to death on King William Island, where indigenous Inuit had lived successfully for over 700 years (Boyd et al., 2013). Other examples include the 1860 Burke and Wills expedition in Australia or the Narvaez expedition in what today is Florida. The last two examples are particularly interesting because they show that an exception to the standard story appears to only occur when the lost European explorers engage in cumulative cultural evolution, learning the culture of local populations. In the case of the Burke and Wills expedition, at one point the explorers were saved by indigenous hunter-gatherers, who showed them how to make bread from a seed called 'nardoo,' which could be pounded, made into flour, and baked as a bread. However, the explorers didn't follow the indigenous cultural practices of preparing the nardoo precisely. They did not leach the flour with water extensively, they did not expose the flour to ash during heating, and they did not eat the nardoo from mussel shells. It turns out that each of these was an important detoxification practice that combats the high levels of thiaminase in nardoo. In the end, despite the presence of abundant food around them, the Burke and Wills both died. There was one additional person, named King, on the expedition who survived long enough to be saved. How did he do it? By living among the local population. In other words, by completely relying on their cumulative cultural knowledge (Henrich, 2016, pp. 27-30). In the Narvaez expedition, of the 300 original conquistadors that were part of the expedition, four individuals were able to survive, but again, this was only because they lived among the local indigenous population (Henrich, 2016, pp. 30-31).

Another source of evidence for the benefits of cumulative cultural evolution is from experiments intended to test for the benefits of this mechanism. Muthukrishna, Shulman, Vasilescu and Henrich (2014) designed an experiment where participants are asked to undertake a difficult task that they have no prior experience with. The experiment was designed to mimic the process of cumulative cultural evolution and to vary its presence. All experiments had 10 generations and information sharing across generations. The experiment was incentivized so that participants' payoffs were increasing in their performance and the performance of subsequent generations. In one treatment, which was intended to model greater access to cumulative cultural evolution, participants in generations 2 to 10 had access to guidance from all five participants in the previous generation. In the other, which was intended to model less cumulative cultural evolution, the participants only had access to information from one of the five participants of the previous 
generation.

There were two versions of the experiment, each with a different task. In one, participants had to create an image using software on a computer. The information that was transmitted between generations was the created image and two pages of written notes/tips from one or five participants (depending on treatment) from the previous generation. In the other, participants had to tie a system of knots using rock-climbing equipment. In this experiment, participants had access to an instructional video created by one or five participants from the previous generation.

The authors find strong evidence that performance on the tasks was greater in the versions of the experiment with more cumulative cultural evolution. These participants had more information when starting and therefore didn't need to 'reinvent the wheel.' In this setting, learning from an accumulated body of knowledge helped them avoid common pitfalls and to gain important insights more easily than if they were learning on their own.

As I will discuss below, a number of findings within the economics literature regarding puzzling aspects of technology and innovation provide evidence in support of models of cumulative cultural evolution. Or put differently, models of cumulative cultural evolution provide a useful framework that can help economists make sense of the process of innovation. I return to this in Section 3 B.

\section{Insights from a Recognition of History as Evolution}

I now turn to a discussion of how an evolutionary framework provides a range of insights that are relevant to economics. At this point, a few caveats are in order. Although I have organized these insights into subsections, the ideas do not necessarily flow from one subsection to the next. These should be thought of as disparate insights that have come to me from reading the cultural evolution and economic history literatures in tandem. In addition, in no way do I think that the insights and connections described below are complete or even representative. We are so early in our thinking of history as evolution that at this times these are a few random points. My sense is that they are still just the tip of the iceberg in terms of fruitful ideas and research that will emerge from a more evolutionary perspective within economics. 


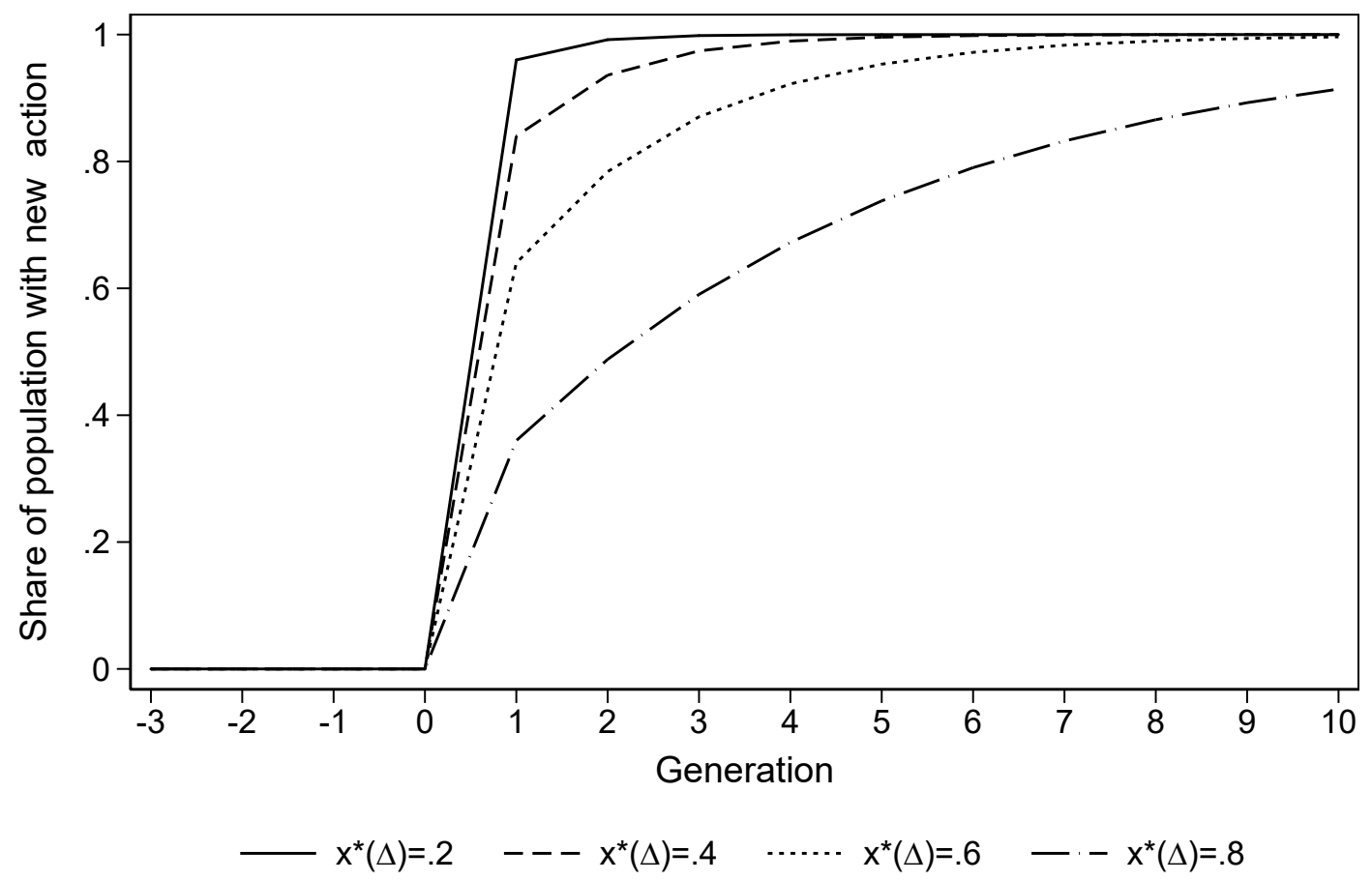

Figure 2: The adoption of a new (optimal) action following a shock depends on the proportion traditionalists in the society $x^{*}$.

\section{A. Environmental Mismatch}

An important consequence of cultural evolution is that it can result in environmental mismatch. To see this, we return to the model of Rogers (1988). In the model, the benefit of culture is that it saves on information acquisition costs. However, the cost is that when the environment changes, traditionalists (i.e. those who rely on culture for decision making) do not choose actions that respond as accurately to the environment as non-traditionalists do. To see this consider Figure 2, which shows how the actions chosen in a society respond to a change in the state of the world, which is assumed to occur between periods o and 1. Different paths are reported, each for a society with a different equilibrium proportion of traditionalists (which is due to differences in the underlying $\Delta$ ). As shown, societies with a lower proportion of the population of traditionalists (low $x^{*}$ ) more quickly and fully respond to the change in the environment by changing the action.

In the model, many individuals continue to choose the old action after the state changes (after period o). This is an example of environmental mismatch. These individuals are choosing an action that is optimal for a past environment but not for the current environment. Because of the slow-moving nature of culture, when actions are chosen based on culture, episodes of mismatch 
can occur.

The most well-known examples of mismatch are actually from evolutionary biology. One is sea turtles. The mothers leave the ocean, come to shore, and bury their eggs on sandy beaches. Once the sea turtles hatch, they need to be able to make their way back to the ocean. They have evolved a method that allows them to do this simply: After they are born, at night, they head directly towards any bright light. In their natural environment, the only bright light is the reflection of the moon off of the water. By moving towards the moon's reflection, sea turtles navigate towards the water (Ehrenfeld and Carr, 1967). This evolved mechanism worked extremely well until the environment changed. In the modern world, where cities and freeways with bright lights are often located next to beaches, this biological heuristic works less well. Instead of heading towards the ocean, they move towards city lights which are in the opposite direction of the ocean (Salmon, Tolbert, Painter, Goff and Reiners, 1995). This is an example of mismatch. A trait that worked well in the environment in which it evolved works poorly in the new environment.

Another commonly cited example is the dodo bird (raphus cucullatus), which is a bird that lived on the Island of Mauritius. A sketch of the bird from 1626 is shown in Figure 3. Because of a scarcity of berries and other food during certain times of the year, dodo birds developed accumulations of fat on its body. They lost the ability to fly but developed a keen sense of smell which allowed them to track down the limited amounts of berries that existed during seasonal scarcity. Because there were no predators on the islands, they didn't develop and special strategies to hide or protect their eggs. Overall, they were well-adapted to their environment (Claessens, Meijer and Hume, 2015, Gold, Bourdon and Norell, 2016). However, after human contact, predators like pigs, rats, and dogs were brought to the islands. The unprotected eggs and the flightless birds did not far well and the species soon became extinct.

The notion of mismatch provides a framework that helps better understand the world and make better sense of recent empirical findings within historical economics. For example, the finding that Africa's slave trades reduced contemporary levels of trust from Nunn and Wantchekon (2011), combined with the evidence that increased trust is associated with higher incomes at both the country and individual levels (Algan and Cahuc, 2010, Butler, Giuliano and Guiso, 2016), suggests that the current levels of trust within Africa may be suboptimal. The current levels of trust may be well-suited to the 400-year period of intensive slave raiding that the continent experienced, but they may be lower than optimal in the current environment. 


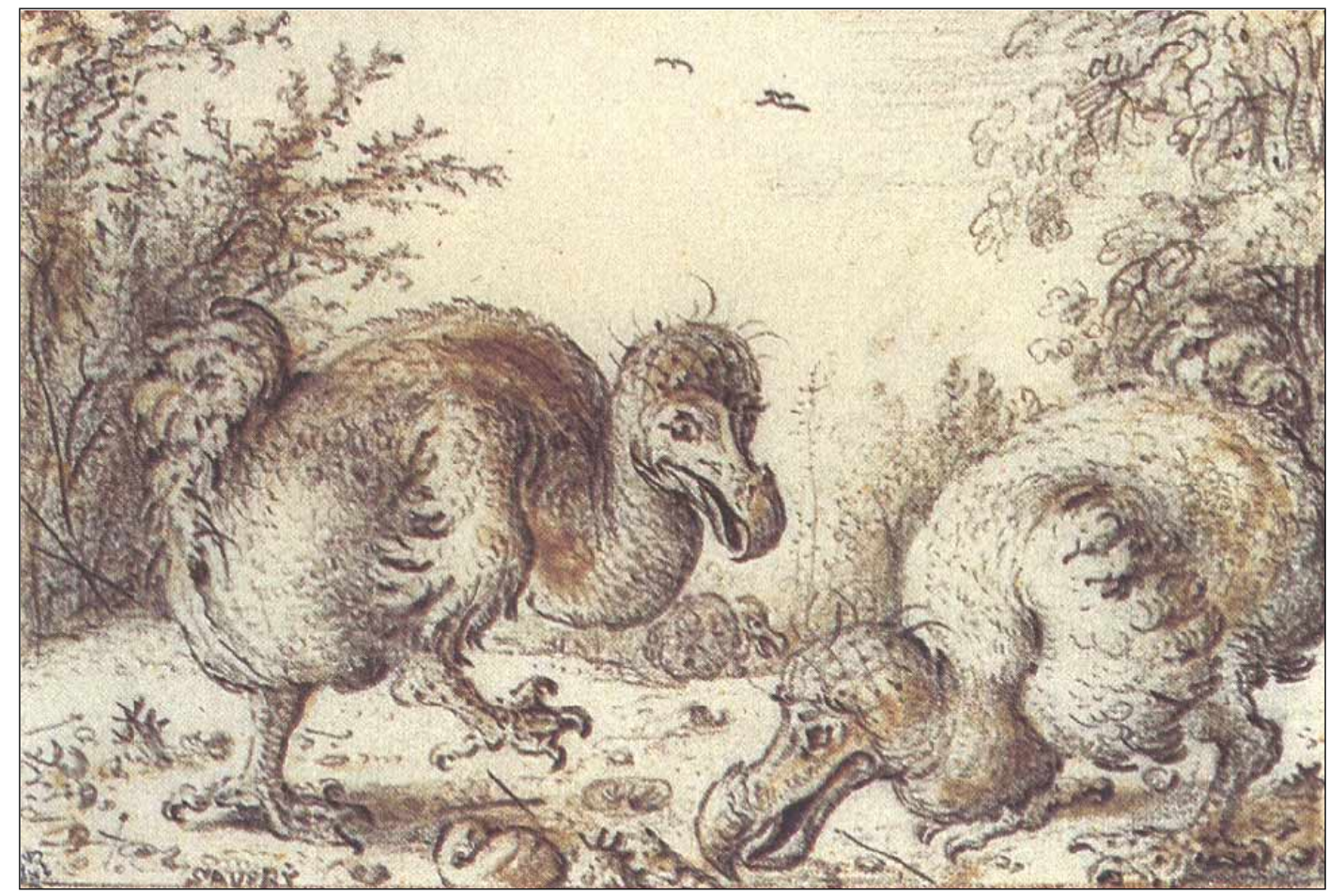

Figure 3: An example of environmental mismatch: The dodo bird (raphus cucullatus). Sketch by Roelandt Savery, 1626. Source: Figure 10 of Hume (2006).

One of the best developed historical examples of mismatch is from Avner Greif's (1989, 1993, 1994) studies of the Maghribi and Genoese merchants of the Medieval Mediterranean. One group, the Maghribi traders, were Jewish traders who had migrated from Baghdad to Tunis and had adopted the values of Muslim society. They began trading in the early 11th Century. According to Greif, their merchant agent relationships relied on information sharing. If an agent cheated a merchant, then no other merchants would hire the agent. This created a form of collective punishment, which required information sharing and the formation of dense information networks. The other group of traders in the region at the time were the traders from Genoa who did not engage in the same form of collective punishment of cheating agents. Instead, the merchants were highly individualistic and did not engage in information sharing.

The wage that had to be paid to keep an agent from cheating was lower in the collectivist regime of the Maghribi than under the individualistic regime of the Genoese. ${ }^{8}$ However, from the early 11th to 12th Centuries, trade between Spain and Constantinople expanded significantly. In response, the Genoese expanded by creating new merchant-agent relationships with

\footnotetext{
${ }^{8}$ In addition, the Maghribi system also featured other benefits. Merchants could use each other as agents. If a merchant-agent was dishonest, then other merchants could punish the merchant-agent by not punishing agents who had cheated that merchant. This additional threat meant that the efficient wage need to pay a merchant-agent to keep them from cheating was even lower (Greif, 1994).
} 
non-Genoese. A consequence of this is that formal organizations and legal practices developed to facilitate these forms of cross-group exchange. The institutions that emerged were helpful for longer-term economic development (Greif, 1994). By contrast, the Maghribi, because of the equilibrium they were in, could only expand by creating new merchant agent relationships within their own group. Thus, more formal institutional structures did not develop and commerce continued to rely on informal enforcement mechanisms such as social norms of group punishment.

Another example of mismatch is highlighted by the recent findings from Alesina, Teso and Stantcheva (2013a), which measures perceptions of intergenerational mobility in Sweden, Italy, France, the U.K. and the United States. They show that the sample from the United States has, by far, the most optimistic perceptions of the amount of economic mobility in their country. This is particularly striking since the United States has the lowest mobility of the countries studied. In addition, for the other countries, the measures of perceived mobility and actual mobility are pretty similar. For the United States, perceived mobility is far from actual mobility. Thus, the United States appears to be a clear outlier in terms of its perceptions about mobility.

While this has yet to be studied thoroughly, the origins of this misperception are most likely due to the fact that in the 19th Century, the United States was a settler economy with very high levels of mobility, and much higher than other countries, like the U.K. at the time (Long and Ferrie, 2013). It was likely this environment that generated some of the values and beliefs that are particularly American, such as a belief in the American Dream (anyone can make it if they work hard enough), a desire to have limited government, and a limited set of policies that economically support the population and/or redistribute income, such as universal health care or high-quality public schooling. While other factors are clearly important, such as the history of race relations, a key determinant of these beliefs is likely the high mobility experienced in the U.S. historically. While these beliefs may have been accurate and well-suited for the historical setting, it is less clear that they are well-suited to the current environment.

Viewing the world through the lens of mismatch also generates insights that are important for policy. One example of this is the recent paper by Heller, Shah, Guryan, Ludwig, Mullainathan and Pollack (2017), which examines the effectiveness of a series of interventions from 2009 to 2015 aimed at improving the outcomes of disadvantaged youth from Chicago. One was a one-year program called 'Becoming a Man' (BAM), which was developed by the Chicago nonprofit Youth Guidance. The other was the same program, but stretched out over two years. The programs 
comprised 2,740 and 2,064 randomly-chosen youth, respectively. Both versions of the program had significant effects. They reduced total arrests by $28-35 \%$, arrests for violent crime by $45-50 \%$, and arrests for other crimes by $37-43 \%$. The authors also found persistent effects on schooling outcomes: graduation rates increased by 6-9 percentage points. The third program had many of the same elements of BAM but was implemented among high-risk juvenile arrestees and was implemented by the Cook County, Illinois Juvenile Treatment Detention Center. This program was also very successful, reducing readmission rates to the detention center by $21 \%$.

The authors also study potential mechanisms that could explain the results. They find that the evidence points to one aspect of the interventions being particularly important. To understand the mechanism, we must first recognize that much of our behavior is driven by automatic impulses - what Daniel Kahneman (2011) calls 'system 1'. (For cultural economists and those studying cultural evolution, system 1 would be associated with transmitted cultural values and beliefs.) This is also true for the youth in the programs, who are from distressed neighborhoods where being aggressive and fighting is often necessary to save one's reputation. However, these automatic responses, although generally adaptive to the youth's environment, may not be the best response in many situations, like in school. The programs helped students develop the mental tools necessary to switch from an automatic reaction based on system 1 responses to one that is more thoughtful, taking into account the specifics of the situation and relies on system 2. According to the authors, the experiments leverage what is known about individual culture and psychology to develop what they call "a greater sense of occasion" (Heller et al., 2017, p. 6).

This example illustrates that one solution to cultural mismatch is to attempt to reduce the reliance of decision-making on the cultural trait. In this case, there was a reduction in the reliance on system 1 and an increase in the use of system 2. The study also provides an excellent example of how knowing the cultural and psychological roots of behavior can help design policy that can effectively improve the actions and outcomes of those involved.

Another example of mismatch and its implications for policy is explored in the recent study by Bursztyn, Gonzalez and Yanagizawa-Drott (forthcoming). The authors study Saudi Arabia, a setting that is much less supportive of female employment outside of the home than the rest of the world. The authors study a sample of 500 married men, aged 18-35, from Riyadh, Saudi Arabia. To qualify for the study, participants had to have a cell phone and at least some college education. 
Participants were divided into groups of 30 individuals from the same neighborhood. They were then asked whether or not they agree that "women should be allowed to work outside the home," as well as their guess about the number of members of their group who hold this view. The authors found that individuals systematically underestimated the support for women working outside the home. This mismatch between beliefs of the norms held by others and actual norms in the population can be understood as a form of mismatch. Within Saudi Arabia, in recent decades beliefs about women's work have been changing. ${ }^{9}$ Thus, beliefs about norms, which evolved in a previous environment, are not accurate in the current environment.

The authors then test whether the perceived and actual norms of others can be more-closely aligned by providing participants with information about the reported survey-based beliefs of others within their group. For a randomly-selected half of the sample, this was done. They find that the information provision increased the likelihood that participants signed up their wives for a job-matching service, and that their wives had applied for a job and had interviewed for a job 3-5 months after the experiment. Not surprisingly, the effects are greater the greater was the gap between the perceived and actual norms. Overall, the study documents the presence of mismatch and shows how information provision can quickly reduce the mismatch.

\section{a. Endogenous Mismatch}

As the examples of mismatch above highlight, a shortcoming of the traditionalist strategy that relies on culture is that the actions chosen by individuals do not trace the environment as precisely as they could. In other words, they generate the potential for environmental mismatch.

Recent research on the origins of the Industrial Revolution can be viewed through the lens of mismatch where the change in the environment is endogenous to the strength of tradition in society. To see this, we again return to the model from Rogers (1988). In the model, the stability of the environment $\Delta$ was exogenously given. However, in reality, there are many examples of the external environment - such as economic conditions, politics, technology, etc - being determined by human actions. Further, the rate of technological innovation, economic growth and political change, itself, can be endogenous to the tradition in society.

\footnotetext{
${ }^{9}$ For example, according to the Global Gender Gap Index, the 'economic participation and opportunity' score for Saudi Arabia increased from 0.24 in 2006 to 0.38 in 2020. In addition, if one looks at the data for Saudi Arabia from the Arab Barometer, one finds strong evidence that individuals born in more recent years have views that are more supportive of women's employment.
} 
Joel Mokyr (2018), in his book Culture of Growth, argues that a crucial determinant of the 18th Century Industrial Revolution in Western Europe was the novel belief that it was acceptable for younger generations to question the wisdom of the previous generations. This change in thinking resulted in a cultural belief that it is possible and desirable to understand how the natural world works, led to innovation and knowledge creation, which ultimately created the economic productivity gains of the Industrial Revolution. Mokyr (2018) argues that the presence of this new cultural trait - a weakening of the importance placed on traditional ways of thinking - was present in Western Europe but not China, which explains why, despite similar levels of economic development, the Industrial Revolution did not occur in China. He argues that "the heavy hand of the respect for the 'ancients' was felt through much of Chinese history" (p. 298). According to this argument, a weakening of tradition, and the resulting cultural change, are key determinants of the Industrial Revolution and the World's current economic prosperity.

Within this model, this means that in addition to $\Delta$ affecting $x^{*}, x^{*}$ can also affect $\Delta$. We can extend the Rogers model to incorporate this mechanism by assuming that the rate of change $\Delta$ is decreasing in the proportion of traditionalists in the economy $x: \Delta(x)$ and $\Delta^{\prime}(x)<0$.

We can also add technological progress to the model by altering the payoffs such that the payoff to a matching state and action is $\pi+\beta$ (rather than $\beta$ ) and to a mismatching state and action is $\pi-\beta$ (rather than $-\beta$ ), where $\pi$ can be thought of as the level of technology in the society. Following the logic of Mokyr (2018), assume that increases in $\pi$ occur as a by-product of the information acquisition of non-traditionalists. Therefore, the growth of $\pi$ is a decreasing function of the proportion of traditionalists in the economy: $\frac{\dot{\pi}}{\pi}(x)$ and $\frac{\dot{\pi}^{\prime}}{\pi}(x)<0$. Further, assume that instability is driven solely by technological innovation and, therefore, instability is increasing in the rate of technological change: $\Delta\left(\frac{\dot{\pi}}{\pi}(x)\right)$ and $\Delta^{\prime}\left(\frac{\dot{\pi}}{\pi}(x)\right)>0$. From this it follows that instability $\Delta$ is a decreasing function of the prevalence of tradition $x$ in society: $\Delta(x)$ and $\Delta^{\prime}(x)<0$.

This setting gives rise to multiple stable equilibria. To see this, first consider an equilibrium where the proportion of traditionalists is high. As a consequence, there is little technological change and the environment is stable. Because of the stability of the environment, the relative benefit of tradition is high, which sustains the high proportion of traditionalists. Thus, such an equilibrium is stable. Second, consider an equilibrium where the proportion of traditionalists is low (even zero). In such an equilibrium, there is a lot of trial-and-error learning, which generates rapid technological change and a highly unstable environment. This in turn results in a relatively 
low benefit of tradition, which sustains the low proportion of traditionalists. Thus, such an equilibrium is also stable.

To see this more formally, recall the payoffs of the two types:

$$
\begin{aligned}
\Pi^{T} & =\pi+\frac{\beta(1-x)(1-\Delta)}{1-x(1-\Delta)} \\
\Pi^{N T} & =\pi+\beta-\kappa .
\end{aligned}
$$

Assume that the probability that there is no shock in a generation, $1-\Delta$, is given by the following function: $1-\Delta(x)=x^{\theta}$ for $\theta \in[0,1]$. With this, the expected payoff of a traditionalist then becomes:

$$
\Pi^{T}=\pi+\frac{\beta(1-x) x^{\theta}}{1-x^{\theta+1}}
$$

The payoffs of the two types are shown in Figure 4 for the case where $\theta=1 / 2 .{ }^{10}$ The payoffs to non-traditionalists remain unchanged since they do not depend on $\Delta$. The payoffs to traditionalists are now increasing in the proportion of traditionalists $x$. This is because the higher the proportion of traditionalists in the economy, the less innovation, the more stable the environment, and the more beneficial is tradition. From the figure it is also clear that the level of technology, $\pi$, does not affect the equilibrium. This is because $\pi$ affects the payoffs of both types symmetrically and additively.

The figure also shows that with endogenous $\Delta$ one can have multiple equilibria. In one equilibrium, $x^{*}=1$. Since the proportion of traditionalists is high, economic growth is low, and the state is stable. This can be thought of as a traditional low-growth equilibrium. In the other equilibrium, $x^{*}=0$. Since the proportion of traditionalists is low, there is rapid economic growth, and the state is less stable. In the figure $x^{B}$ denotes the boundary of the basins of attraction of the two equilibria. Any initial population distribution of traditionalists greater than $x^{B}$ eventually converges to the $x^{*}=1$ equilibrium. Initial population distributions less than $x^{B}$ converge to the $x^{*}=0$ equilibrium.

A necessary condition for multiple equilibria to arise is that the cost of information acquisition, $\kappa$, must be sufficiently high; namely, $\kappa>\frac{\beta \theta}{1+\theta}$. If this is not satisfied, then the equilibrium with $x^{*}=0$ is unique. Intuitively, if acquiring information is sufficiently easy, then traditionalists are not present in equilibrium.

\footnotetext{
${ }^{10}$ The payoffs shown are for the scenario where information acquisition costs are sufficiently high; namely, $\kappa>\beta / 3$ (or $\kappa>\beta \theta /(1+\theta)$ in the more general case). This condition is discussed further below.
} 


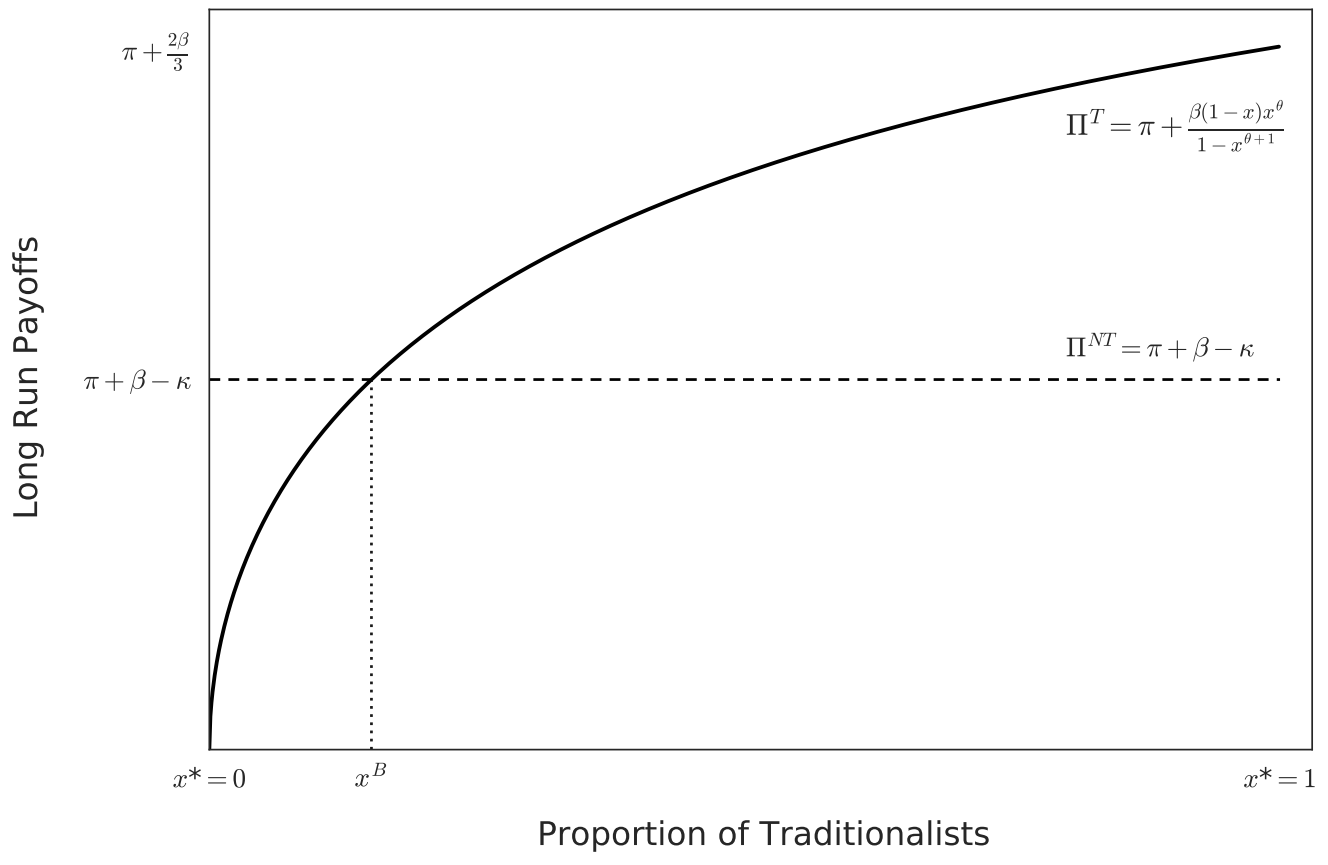

Figure 4: The equilibrium proportion of traditionalists (T) and non-traditionalists (NT), assuming the instability of the environment is endogenous to learning by NT (and $\theta=1 / 2$ ).

Mokyr's description of the industrial revolution is one where society transitioned out of the the $x=1$ equilibrium, with no (or little) information acquisition and a very strong importance placed on following the beliefs and actions of previous generations. Because of the strong reliance on tradition, the external environment is stable, which means a reliance on tradition is efficient.

In this setting, a society transitioning from the former to the latter equilibrium requires a reduction in the number of traditionalists below $x^{B}$. Once this occurs, economic growth increases and the environment becomes less stable, which further reduces the benefits to tradition.

\section{B. Education and Cultural Evolution}

I now turn to a description of how cultural evolution can be used to provide a deeper and more realistic understanding of education, human capital, and innovation. To see this, I turn to the definition of culture. Within the cultural evolution literature, the standard definition is something along the following lines: "[culture is] the transmission from one generation to the next, via teaching and imitation, of knowledge, values and other factors that influence behavior. Cultural transmission may have a variety of structures... For example, parents may enculturate 
their offspring or peers may enculturate each other." (Boyd and Richerson, 1985, p. 2). This definition is one that is very similar to the typical definition that has been adopted within the field of cultural economics. For example, Guiso, Sapienza and Zingales (2006) have the following definition "customary beliefs and values that ethnic, religious, and social groups transmit fairly unchanged from generation to generation." (p. 23).

At first glance, the two definitions of culture appear essentially identical, which is not surprising since the cultural economics literature builds upon the cultural evolution literature. Both definitions describe the transmission of values and beliefs from one generation to the next. ${ }^{11}$ A small but important difference is that "knowledge" and its transmission is mentioned in the definition of Boyd and Richerson (1985) but not Guiso et al. (2006). This is a huge difference in how culture is defined and conceptualized in economics compared to evolutionary anthropology. Within economics, the accumulation and transmission of knowledge within or across generations is not culture. That is human capital. When I first came across the anthropological definition, my immediate reaction was likely the same as the reaction you are likely having now. Knowledge and technology are conceptually very different from culture. They are examples of human capital and not of culture. But, I have come to realize that generally the difference is not clear and making a conceptual distinction between the two is problematic and not particularly helpful.

Take for example knowledge of how to create arrows that are used for hunting. Henrich (2016) describes this process for the indigenous hunter-gatherers of Tierra del Fuego. This production process is relatively simple, requiring only fourteen steps and six material inputs. Henrich (2016, p. 107) describes some of the steps and I quote him directly:

- The process begins by selecting the wood for the shaft, which preferably comes from chaura, a bushy, evergreen shrub. Through strong and light, this wood is a nonintuitive choice since the gnarled branches require extensive straightening. (Why not start with straighter branches?)

- The wood is heated, straightened with the craftsman's teeth, and eventually finished with a scraper. Then, using a preheated and grooved stone, the craftsman presses the shaft into the grooves and rubs it back and forth, pressing it down with a piece of fox skin. The fox skin become impregnated with the dust, which prepares it for the polishing stage. (Does it have to be fox skin?)

\footnotetext{
${ }^{11}$ It is true that the definition of Guiso et al. (2006) does not mention transmission between those of the same generation (horizontal transmission). However, it is clear that the cultural economics literature does not exclude this form of transmission.
} 
- Bits of pitch, gathered from the beach, are chewed and mixed with ash. (What if you don't include ash?)

- The mixture is then applied to both ends of a heated shaft, which must then be coated with white clay. (What about red clay? Do you have it heat it?) This prepares the ends for the fletching and arrowhead.

- Two feathers are sued for the fletching, preferably from the left wing of the bird, and vise versa for the lefties. (Does this really matter?)

- The feathers are lashed to the shaft with sinews from the back of the guanaco, after they are smoothed and thinned with water and saliva. (Why not sinews from the fox that I had to kill for the aforementioned skin?)

Learning how to successfully create arrowheads requires years of apprenticing. The knowledge, much of which is tacit, is taught over an extended period of time and much of it is codified in terms of tradition. The underlying mechanics and reason that a certain feather is used or a certain wood is not understood. Instead, the learning is of culture and traditions. In this case culture is synonymous with knowledge and human capital. As we will discuss further below, one actually "learns" more by adopting the traditions of one's culture.

There are numerous examples where the accumulation of human capital is really cultural transmission - i.e., information and knowledge that is transferred through cultural learning. Such examples are also present within the economics literature, although this is often referred to as 'tacit knowledge' acquisition rather than cultural transmission. One example is the famous case of Desh Garments, where as part of a 1980 joint venture agreement between South Korea's Daewoo and Bangladesh's Desh garments, 130 workers from Desh garments were brought to a Daewoo garment plant located in Busan, South Korea (Easterly, 2001). This episode of cultural transmission had a dramatic effect on garment production in Bangladesh. Annual production increased from 43,000 shirts in 1980 to 2.3 million in 1987. Of the 130 Desh workers who had traveled to South Korea, 115 of them left Desh garments to start their own garment companies at some point in the 1980 .

Another example, but in a more controlled setting is from an experiment that intentionally varied the amount of cultural transmission to textile firms in India (Bloom, Eifert, Mahajan, McKenzie and Roberts, 2013, Bloom, Mahajan, McKenzie and Roberts, 2020). The authors study 17 firms 
that comprise 28 medium-sized (100-1,000 employees) family-owned textile plants, located in Maharashtra, India. Fourteen of the 28 plants received five months of extensive management consulting, which was valued at \$250,000. The consulting was intended to improve management and operations within the plants. They found that in the months following the study, the treatment resulted in fewer defects, inventory savings, and higher total factor productivity. Nine years after treatment, they found that although about half of the previously adopted management improvements had been abandoned, the treated plants still were much more productive than the non-treatment plants. In addition, they found additional forms of cultural transmission. The practices had fully spread to non-treatment plants (even those not involved in the experiment at all) that belonged to the same company (i.e., firm) as a treatment plant. Thus, cultural knowledge was transmitted fully within the company.

A theoretical model of such processes of cultural transmission was developed by Henrich (2004b). To anthropologists, the model is one of cultural transmission. To economists, it is one of human capital accumulation. In the model, each period there is a role model. Depending on the setting, this person could be a master craftsman, the village sage, or even a professor of economics. There are $N$ pupils who learn from the role model, who was the most prolific and highly skilled individual of their generation. After learning from the master, the cultural knowledge / human capital of the pupils is determined from a draw from a distribution. The person with the highest draw then becomes the master who conveys their knowledge to the next generation.

Let $z_{i}$ denote the level of cultural knowledge and human capital of individual $i$. The role model's $z$ is denoted $z_{\max }$ and is shown by the vertical line in Figure 5. Also shown is the probability distribution of draws of $z$. In the original model, it is assumed that the distribution is Gumbel $(\alpha, \beta)$, but subsequent studies find that the predictions hold for a large class of distributions (Vaesen, 2012). ${ }^{12}$ Because the role model is the individual with the highest skill in the previous generation, as shown in Figure 5, students' $z_{i}$ 's tend to be lower than that of the role model.

Because the state of knowledge of the next generation, as measured by the $z_{\max }$ of the role model, is governed by the maximum draw of $z$ in that generation, a larger population (and

\footnotetext{
${ }^{12}$ Also see Kobayashi and Aoki (2012) for various extensions of the model, including allowing for the more-realistic assumption that role models are chosen from an individual's network of acquaintances.
} 


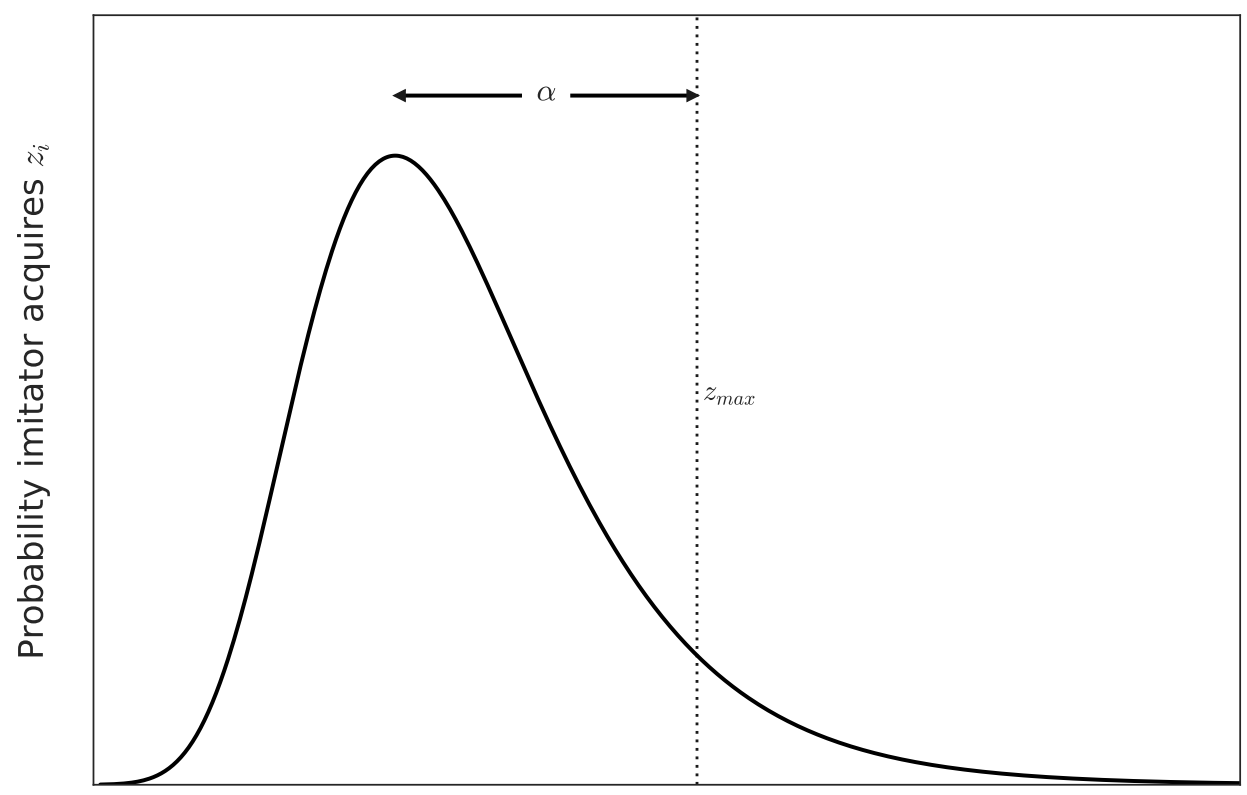

Value of $z_{i}$ acquired by the imitator

Figure 5: Illustration of the model of cumulative culture / human capital developed by Henrich (2004b).

therefore more draws), will result in a higher level of skill for the role model. More specifically, for the Gumbel distribution, the level of skill of the role model in the next period is given by

$$
z_{\max }=\alpha+\beta(\gamma+\ln N)
$$

where $\gamma$ is the Euler-Mascheroni constant, which is approximately 0.5772 . In addition, the growth in the average level of human capital in the economy is given by

$$
\Delta \bar{z}=-\alpha+\beta(\gamma+\ln N)
$$

In other words, for sustained knowledge growth (or cumulative cultural evolution), the population has to be sufficiently large. In addition, if the population is below a threshold size, then it can experience technological regress.

The model has interesting predictions. The rate of knowledge accumulation is increasing in the size of $N$. This is knowledge embodied in individuals' minds and the knowledge of the next generation's master is the max of all $N$ draws. The more draws one has the higher the max will be. The models also show how a society can have technological regress. If $N$ is too small, then the maximum among the $N$ draws can be lower than the skill of the master. Henrich (2004b) uses 
this to explain the technological regress observed amongst the Tasmanians after Tasmania was separated from Australia due to the rising sea levels during the Holocene glacial retreat.

The important point here is that a model of cultural evolution is isomorphic to a model of human capital accumulation. In this sense, given that human capital has been at the core of our discipline for centuries, it turns out that we have all been believers in culture (at least according to the definition of anthropologists) all along.

\section{a. Innovation and the Collective Brain}

To drive home the similarity of cultural transmission and knowledge accumulation, both empirically and theoretically, I will compare two ways of thinking about knowledge. One will be familiar to the reader and is at the center of endogenous growth theory. The other, which will be less familiar, is from evolutionary anthropology and emphasizes the fact that knowledge creation occurs through a process of cumulative cultural evolution and cultural transmission.

As a conceptual framework to aid in the discussion, consider the following setting. Society has a population of size $L$. Assume that the fraction $\gamma$ of the population $L$ is exposed to a role model and thus has the opportunity to innovate. For those with this opportunity, innovation occurs with probability $\mu$. The extent to which an innovation diffuses into the aggregate economy (e.g., through social learning or cultural transmission), increasing the rate of aggregate knowledge growth $\dot{A} / A$, is increasing in the connectivity and cohesiveness of the population $\theta$. Thus, knowledge growth is given by:

$$
\frac{\dot{A}}{A}=\gamma \mu \theta L
$$

This is a highly-simplified version of an endogenous growth model with scale effects (meaning that the rate of innovation is increasing in the population size). However, it can also be interpreted as a representation of the notion of the 'collective brain' from evolutionary anthropology, which we discuss in further detail below (Muthukrishna and Henrich, 2016).

Research within the field of economics has tended to focus on two terms in equation (2). The first is population size, $L$. The (initially surprising) prediction that the rate of technological change should be increasing in the size of the population, $L$, was famously tested and confirmed by Kremer (1993). ${ }^{13}$ The second area of focus has been on the level of human capital (e.g., health

\footnotetext{
${ }^{13}$ Interestingly, this prediction was also confirmed by anthropologists who found a strong positive relationship between population size and the number and complexity of pre-industrial marine tools across Polynesian islands (Kine and Boyd, 2010).
} 
or education) which is a primary determinant of $\mu$ within the expression (Mankiw, Romer and Weil, 1992).

While research within cultural evolution has also studied the importance of scale effects (e.g., Derex, Beugin, Godelle and Raymond, 2013), it has also emphasized the importance of parameters associated with cultural transmission of ideas; namely the extent of connectivity and social learning within a population: $\gamma$ and $\theta$. The parameter $\gamma$ captures aspects of a society that influence the extent to which all individuals are able to participate in the innovative process. Inclusivity can be along the lines of gender, socioeconomic status, race, ethnicity, place of birth, etc. The parameter $\theta$ can be thought of as capturing how cohesive or connected a population is, which facilitates the diffusion of existing knowledge and new innovations. Within the literature, this is viewed as the first-order determinant of a society's success. As Joseph Henrich (2004b, p. 214) puts it: "If you want to have cool technology, it's better to be social than smart." The focus on these determinants of aggregate knowledge, which are inherently social, has been developed within a conceptual framework called the 'collective brain' (Muthukrishna and Henrich, 2016). Within this framework, the key to knowledge creation is cumulative cultural evolution and social learning. This is what effectively allows humans to have access to a larger repertoire of knowledge and technology that could ever fit into any one individual's brain. This larger network of knowledge is our 'collective brain'.

This perspective provides insight into findings from recent research within economics that seeks to understand the determinants of innovation within the United States. Bell, Chetty, Jaravel, Petkova and Van Reenen (2019) use data from 1.2 million inventors linked to tax records to document the determinants of innovation within the United States, paying particular attention to factors related to the childhood environment. They document significant variation across the United States, with the U.S. South having particularly low levels of innovation. While a (modest) portion of this is explained by educational attainment, they find other factors to be particularly important, including race, gender, and parental income. Interestingly, these effects are most pronounced for the most highly educated children, suggest that education may be a necessary but not sufficient condition for innovation. There appears to be a missing ingredient that is needed beyond education and that is correlated with observables like race, income, or residential location.

The study then attempts to better understand this missing ingredient. Although they do not 
use this terminology, the patterns they find are exactly those that are predicted by models of the 'collective brain' that feature cumulative cultural evolution. They find that the children of inventors are more likely to be inventors themselves. This itself is not particularly surprising, but they also find that the innovation of the children tends to occur within the same fine-grained technology class as their father. The most likely explanation for this is role-model effects and the transfer of cultural knowledge from fathers to sons. The evidence is not consistent with this being the intergenerational transmission of more general human capital or knowledge which is more relevant for certain technology classes. Child innovation plummets drastically within technology classes that are only one or two classes away from those of the parents' innovations. Thus, this is consistent with specific knowledge being transmitted from parents to children.

This finding is perhaps surprising when one is working within the traditional economics view of innovation where an innovator is tinkering in their garage and come up with a new invention. However, the collective-brain framework has cumulative culture and social learning at its core. Thus, innovation, which is cumulative, is impossible if one is not first exposed to ideas, beliefs, values, and mental models. In addition, since vertical transmission of culture from parents to children is the core mode of cultural transmission, it is not surprising that a child's ability to innovate depends on the cultural knowledge of their parents. As Muthukrishna and Henrich (2016, p. 4) put it: "The most basic structure of the collective brain is the family. Young cultural learners first gain access to their parents, and possibly a range of alloparents (aunts, grandfathers, etc.)."

Bell et al. (2019) also test for the importance of another form of cultural transmission oblique transmission - although the authors do not use this terminology and instead refer to this as transmission from "parents' coworkers to children". They calculate the patent rate among workers in the father's (NAICS 6-digit) industry of employment. They find that the rate of innovation in their father's industry of employment is strongly predictive of child innovation as an adult. In addition, they also find that here too, the child's innovation tends to occur within the same technology class as the innovation by the parents' coworkers.

Although the paper does not have a theoretical framework, the authors' conceptualization of the mechanisms at play is described as follows. "... the data point to mechanisms such as transmission of specific human capital, access to networks that help children pursue a certain subfield, acquisition of information about certain careers, or role model effects." (Bell et al., 
2019, pp. 688-689). In other words, the authors are describing the collective brain model where knowledge occurs through cumulative cultural evolution and social learning.

Complementary evidence for the importance of social learning for innovation can be found in a recent study by Andrews (2019) that looks at the effects of U.S. prohibition against alcohol in the early 2oth Century. Recognizing the importance of bars, taverns and saloons for building network connections that facilitated knowledge flows, he estimates the effects that prohibition, which legally penalized these establishments, had on inventive activity as measured by patenting rates. He finds that after the imposition of state-level prohibition, previously wet counties had $8-18 \%$ fewer patents per year relative to consistently dry counties. Thus, removing these locations of connectivity had sizeable detrimental effects on innovative activity.

The logic of cultural evolution and its connection with technology, innovation, and productivity, helps us to understand the long-term permanent effects that historical events that (temporarily) reduced a population's inclusiveness. An example is slavery within the United States, where under slavery a large proportion of the population was restricted from basic rights and freedoms. Even following the abolition of slavery, intimidation, violence, fear, and discriminatory policies were used to further exclude Americans of African descent. The relationship between slavery and long-term underdevelopment has been well-documented (e.g., Mitchener and McLean, 2003, Nunn, 2008). More specific to the mechanisms of cultural evolution and innovation, Cook (2014) studies the effects of race riots and lynching on the patent rates of African Americans. According to her estimates, these historical events reduced African American patenting by $15 \%$ annually between 1882 and 1940. This is a sizeable effect and its persistence is a likely suspect for explaining the racial differences in innovation documented by Bell et al. (2019). According to the logic of the 'collective brain', when a group is excluded or discouraged from participating in cumulative knowledge creation, this decreases the rate of innovation not only in the current period but also in all future periods.

A potentially important factor affecting a society's level of connection and cohesion $-\theta$ in equation (2) - is generalized trust. There is a strong positive relationship between generalized trust and per capita income. This has been shown to be causal and is found whether one looks across countries, U.S. states or European regions (Algan and Cahuc, 2010, 2013). There is evidence that at least part of this relationship is due to a causal effect of trust on innovation and knowledge creation. Micro-level evidence for this is found in a recent study by Nguyen (2018) that studies 
the CEOs of 3,598 public firms in the United States. She collects information on the ancestry of the $\mathrm{CEO}$ and of all inventors within the firm and finds that across firms, innovation is more rapid and of higher quality if a CEO is from a place with higher levels of trust in general and higher levels of trust in the ancestral origins of the inventors in the firm.

These benefits raise the question of why all countries do not have higher levels of trust than they do. One explanation for this is that historical shocks have been documented to have adverse effects on trust (Nunn and Wantchekon, 2011, Alsan and Wanamaker, 2018, Lowes and Montero, 2017). Alternatively, societies may be stuck in 'distrust traps,' where economic activity is low because trust is low which maintains low levels of trust (Guiso, Sapienza and Zingales, 2008).

A final answer to this is more subtle and stems from the fact that the positive relationship between trust and income is only found at the society level - e.g., regions or countries. When one looks at the individual level, the relationship is actually not positive and monotonic but hump-shaped (Butler et al., 2016). That is, a sufficiently high level of trust is associated with less, and not more, income. Thus, high levels of trust are associated with better outcomes at the society level but not at the individual level. In other words, trust is a prosocial cultural trait and therefore subject to the same trade-off between benefits to the individual and society like other such traits.

Another example of the connection between models of the collective brain and research in economic history can be found in studies of international contact and migration, which can be thought of as ways to increase the size, diversity, and connectivity of the collective brain. Consistent with theory, immigration has been shown empirically to be associated with higher incomes, more innovation, and stronger international business connections, both in the short-, medium- and long-runs (Burchardi, Chaney and Hassan, 2019, Sequeira, Nunn and Qian, 2020). The effects that connectivity can have on economic activity were recently studied by Campante and Yanagizawa-Drott (2018). The authors document a strong effect of the presence of direct flights between cities on foreign ownership links between these locations.

\section{How and Why History Matters}

Another important aspect of cultural evolution is that it is cumulative. As with biological evolution, the benefit of mutations or the optimal 'next step', depends on the current state of the organism and the environment. In addition, progress must be made in a series of incremental 
steps and one is not able to jump to a whole other configuration. Further, each incremental step has to be one that improves the well-being of the organism.

To convey the basic logic of this, Richard Dawkins (1996) in his well-known book Climbing Mount Improbable uses the analogy of climbing hills and mountains. One's location on a slope is the product of a set of cumulative and incremental steps. An additional requirement in an evolutionary setting is that one is only able to move uphill. Since elevation represents the payoff or fitness of an organism, the next step is only taken (i.e., a change is made) if it results in improvement and moves you uphill. Thus, one is not able to move downhill.

An example of this process is provided by Figure 6, which provides a visual metaphor of the evolution of the eye drawn by neurobiologist Michael F. Land (Dawkins, 1996, p. 195). The figure shows a series of peaks, which represent groupings of the known types of eyes that have developed in various parts of the animal kingdom. It is estimated that eyes have independently evolved between forty and sixty times (Dawkins, 1996, p. 139). The figure shows numerous peaks the heights of which provide some sense of how well-functioning the developed eyes are. The lower peaks represent the presence of photocells that detect light by capturing photons with a pigment and translating them into nerve impulses. The highest ranges are the most complex eyes found in the animal kingdom that provide fine-grained and precise eyesight.

Two types of peaks are shown: those on the left that represent compound eyes and those on the right representing camera-type eyes. Camera eyes are the eyes that evolved among many mammals including humans. They comprise light-sensitive photocells forming a cup, a pinhole, and a lens (or a curved mirror, denoted by lower peaks). Another completely different type of eye is the compound eye, which is common among insects, crustaceans, and some worms. The main difference here is in how photocells are organized to receive photons. With camera eyes photo cells are organized as if on the inside of a cup backed by an opaque screen, so that they look inwards to each other. By contrast, with compound eyes, the photocells are placed on the outside of the cup, causing them to look outwards in different directions.

In Figure 6, the different peaks represent different equilibrium outcomes or effective steady states. It is only at these peaks that there is no additional step to be taken. No additional movement can move one further up a slope. The compound eye peaks are drawn to be lower than the camera eyes. This is because compound eyes are not able to see in as much detail as camera eyes unless they are extremely large. For example, for humans to see as well with a 


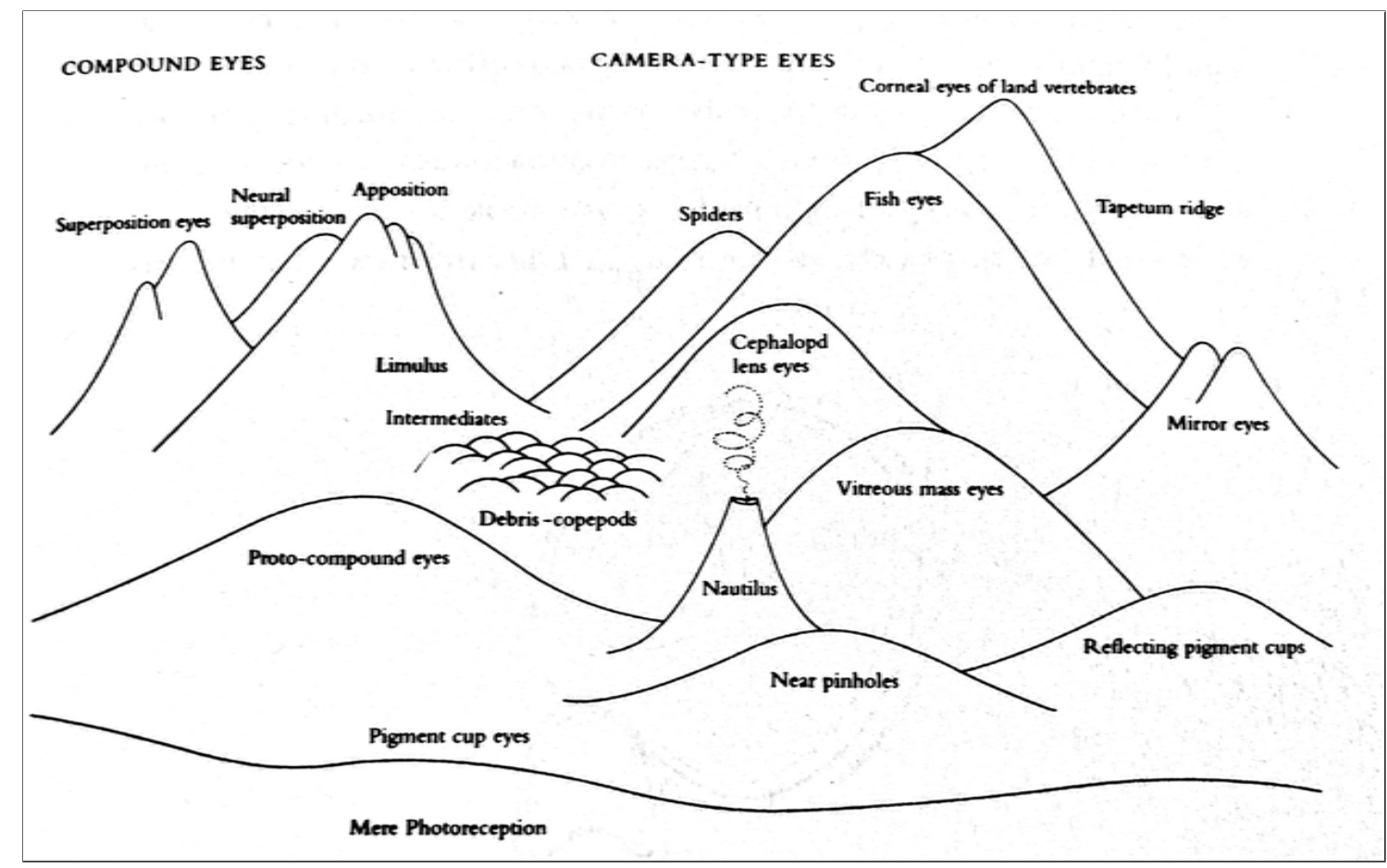

Figure 6: Example of a mountain landscape dictating the evolution of the eye. Source: Figure 5.30 of Dawkins (1996).

compound eye as with our camera eye, the compound eye would have to be 24 meters in diameter (Dawkins, 1996, p. 181).

Now imagine that one is at one of the compound-eye peaks in Figure 6. Can one move to one of the even higher peaks within the camera-type eye mountain range? The answer is no. This would require one to move downhill which is not allowed with evolution. Effectively, there is a valley between the two peaks that cannot be crossed.

At the risk of the 'no-downhill' rule seeming arbitrary, let's look at its logic with a concrete example. One characteristic of camera eyes is that the image that is formed is upside down. With compound eyes, the image is right side up. Thus, if an organism that had compound eyes evolved to have camera eyes, its image-processing nervous apparatus would no longer match its eyes and it would be worse off. Such an innovation would not survive. The only way that a switch could be made is if an animal completely lost its image processing nervous apparatus so that with a switch from compound to camera eyes the organism is no worse off.

The analogy of climbing mountains is overly simplistic since it has reduced evolution to three dimensions. In reality, fitness and payoffs depend on many factors, resulting a setting with very high dimensionality. However, the analogy still provides important insights into path dependence 
and why sometimes it is very difficult to undo previous paths taken.

I now turn from biological evolution to a social setting with cultural evolution. As I discuss, the same logic applies given the nature of culture and its evolution. The example draws from a stylized model from Nunn (2007). The model features two cultural types, those who produce and those who engage in predation, stealing from those who produce. Each type has a bundle of cultural traits, including accumulated experience, that supports the activity. The model assumes that each period a thief can steal from at most one producer and producers can only be robbed once. There is perfect information and no search costs. If a producer is robbed, the thief steals a fraction $q$ of the producer's output, which is given by $A$. We let $x$ denote the fraction of the population with a culture of theft.

The payoffs to the two cultural traits are shown in Figure 7a for different values of $x$. Despite the extreme simplicity of the setting, the model yields multiple equilibria, which are denoted in Figure 7 a by $x_{0}^{*}, x_{B}^{*}$ and $x_{1}^{*}$. When $x=0$, the payoff to the cultural trait of hard work is greater than to the trait of theft and this is an equilibrium, $x_{0}^{*}$. Here, the only cultural trait that exists is one of hard work. There are also two additional equilibria, where both cultural traits exist, and the payoffs to the two traits are equal, $x_{B}^{*}$ and $x_{1}^{*}$.

Now consider the fact that cultural evolution is cumulative and that only innovations that improve one's payoff are adopted. That is, you can only 'walk uphill'. Such a restriction can be micro-founded by assuming that in each period each individual, with some probability $\alpha$, has an experience that potentially changes their cultural values. ${ }^{14}$ As part of this experience, the individual learns the cultural value and payoff of another randomly chosen individual and compares it to their own. If the other person has a different cultural value and a higher payoff is observed, the individual adopts that value with a probability that is equal to $\gamma$ times the difference between the payoffs. As shown by Gintis (1997), given these assumptions one finds that cultural traits grow over time if and only if their average payoffs are higher than the average payoff in the whole population. That is, cultural traits that tend to do better than average grow and those that tend to do worse shrink. Within the model of Nunn (2007), the cultural evolution is given by:

$$
\frac{\dot{x}}{x}=\alpha \gamma\left\{\Pi^{R}(x)-\bar{\Pi}(x)\right\}
$$

\footnotetext{
${ }^{14}$ This assumption is consistent with the existing evidence that events within a person's lifetime, whether it is attending celebrations, watching certain television programs, or shocks to the macro environment, can affect a person subsequent cultural values and beliefs (e.g., Madestam and Yanagizawa-Drott, 2011, La Ferrara, Chong and Duryea, 2012, Giuliano and Spilimbergo, 2014, Cornelson, 2018, Bursztyn et al., forthcoming).
} 


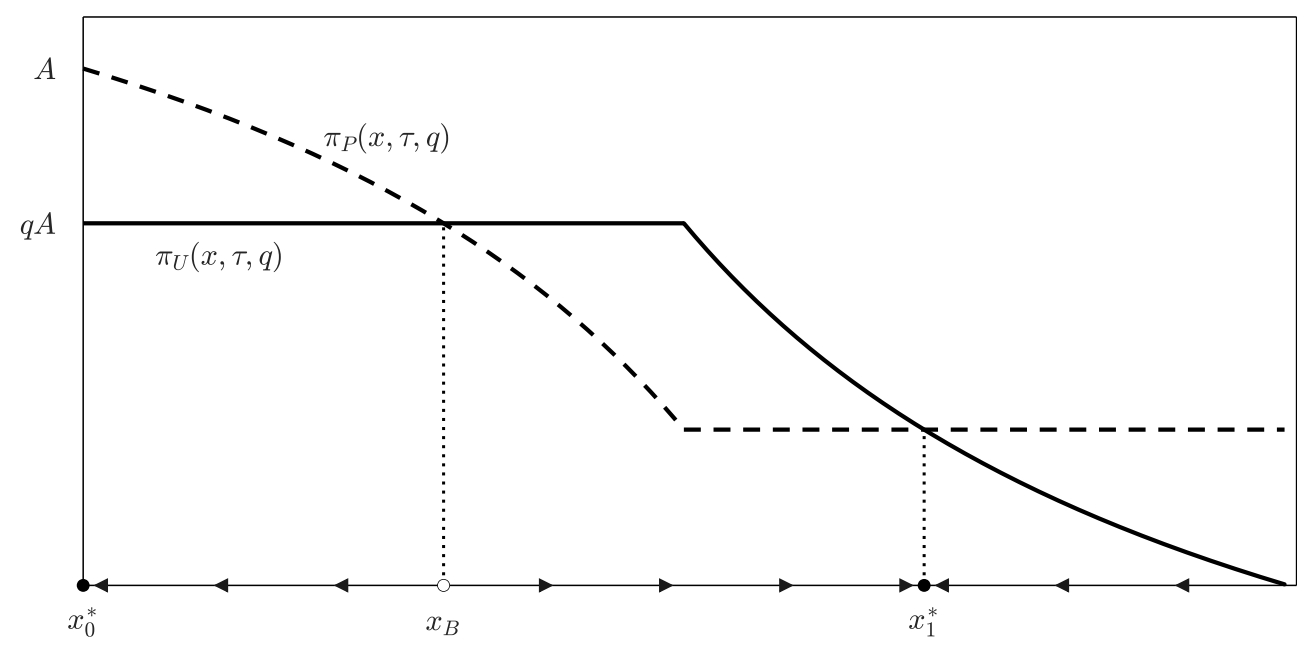

(a) No extraction, $\tau=0$

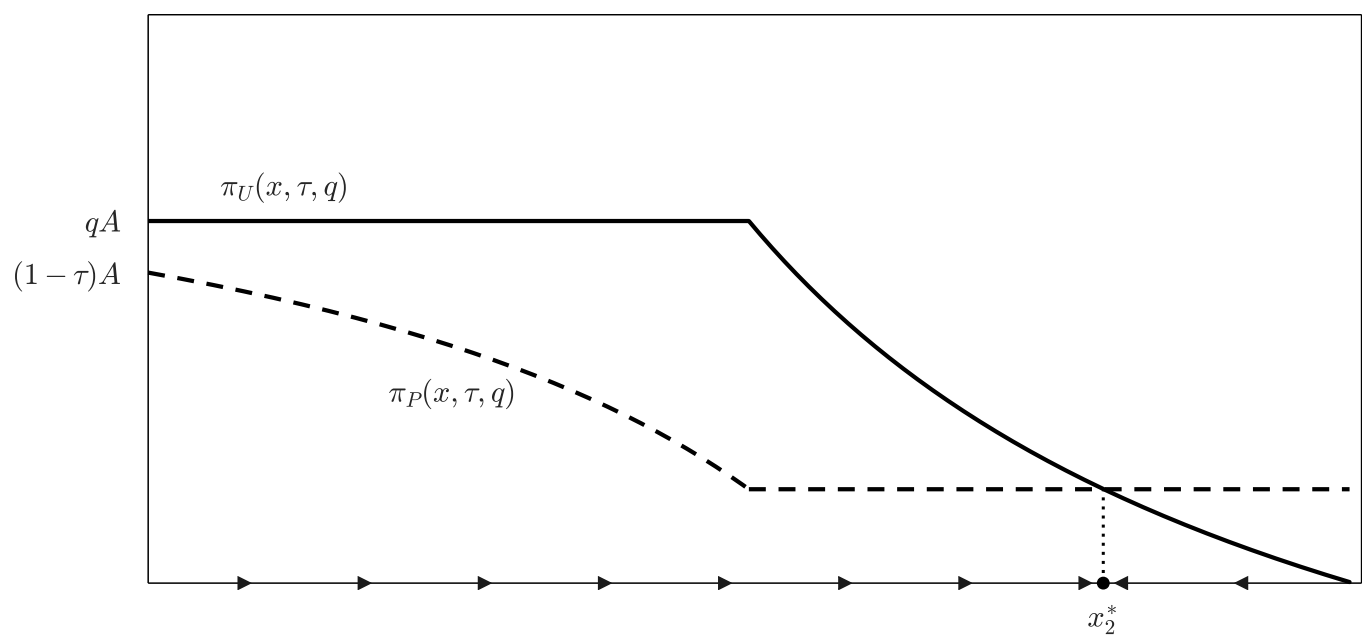

(b) Temporary extraction, $\tau=.4$

Figure 7: Temporary historical events and permanent effects. 
where $\bar{\Pi}(x)$ is the average payoff for the whole population, i.e., $x \Pi^{R}(x)+(1-x) \Pi^{E}(x)$.

Given this dynamic, which formalizes the notion that cultural evolution is incremental and cumulative, a number of insights emerge. The first is that one of the three Nash equilibria above is unstable. This is the equilibrium marked $x^{B}$. It is straightforward to verify that a slight change in $x$ either above or below $x^{B}$ will generate movements in $x$ away from this equilibrium. Thus, $x^{B}$ is not a peak, but actually a valley that divides two peaks.

The second insight the model offers is a deeper understanding of why it is so hard for a society stuck in a socially suboptimal equilibrium to switch to another more socially-beneficial equilibrium. Consider a society, in equilibrium $x_{1}^{*}$. This could describe the low-income situation in many developing countries. Here, average payoffs are comparatively low. Everyone is optimizing. In other words, those at this peak cannot climb any higher. Just as developing countries can see the wealth of rich countries, those at this peak see another higher peak far off in the distance. However, they are not able to get there. Doing so would require individuals to move downhill; namely, adopting behaviors that make them worse off. In this case, this is switching from rent-seeking to production. In popular media, and even journal articles, one regularly observes writers asking the question "why do poor countries stay poor?" Without an evolutionary perspective, particularly one rooted in cultural evolution, this question may appear as a puzzle. However, with this perspective, the reason for this is clear and the question itself seems kind of odd.

Another insight that emerges involves the importance of history. What determines which equilibrium of the two a society is located in? The answer in this setting is history. This is due to the cumulative and incremental nature of evolution. To see this consider two examples. The first is one where there is stability such that no parameters change over the history of the society. In such a setting, the long-run equilibrium is determined by the value of $x$ during the very first time period, $x_{0}$. This uniquely determines the equilibrium in the long-run. If $x_{0}<x_{B}$, then in the long-run the equilibrium is the socially optimal equilibrium where $x=x_{0}^{*}$. If $x_{0}>x_{B}$, then the long-run equilibrium is the socially suboptimal one where $x=x_{1}^{*}$. Thus, history, meaning the historical conditions of a society, can matter for long-term outcomes. Within an evolutionary framework, this fact is obvious. Rather than asking 'why history matters?', we should be asking 'why wouldn't history matter?'.

Next, consider a scenario where the parameters of the model change. Figure $7 \mathrm{~b}$ shows the 
effects of a temporary historical event that reduces the payoffs of those who engage in production relative to those who steal. As shown, the payoffs are tilted so much that the $x_{0}^{*}$ equilibrium disappears leaving a unique equilibrium, denoted $x_{2}^{*}$ where a high proportion of the population has a culture of theft. For a society initially in the $x_{0}^{*}$ equilibrium, over time, in accordance with cultural evolution governed by equation (3), those with a culture of work slowly adopt a culture of theft. This occurs as the society converges to the $x_{2}^{*}$ equilibrium. In other words, the change in the environment caused one of the two peaks to disappear leaving a path upwards to a single peak that is relatively low.

Even if the episode is short-lived and the society has not fully converged to the new equilibrium, as long as the culture of theft has become sufficiently prevalent by the end of the episode, i.e., $x>x^{B}$, then the temporary event will have permanently moved the society from the equilibrium where everyone has a culture of work, $x_{0}^{*}$, to one where most of the population has a culture of theft, $x_{1}^{*}$. Returning again to the analogy, if the trek upwards towards the low peak has progressed sufficiently, even after the high peak returns, it is impossible to get to it. This is because it would require first going downhill to then climb back up. All the society can do is continue upwards to the smaller peak.

This example illustrates another way in which historical events can matter, even if the event are only temporary. Nunn (2007) argues that such a sequence of events potentially explains the long-term effects that colonialism and the slave trade appear to have had on many African societies.

\section{a. Kludges}

In the example above, when one is to the right of $x_{B}$ movement towards the lower-peaked equilibrium $x_{1}^{*}$ as individuals switch from production to theft is making those who switch better off. They are optimizing. However, each subsequent step in this direction, in a sense is making things worse because one is moving away from the highest peak. The further one moves up the path towards the low peak, the harder it is to get back to the high peak (even if one is allowed to move downhill).

Such situations have been termed 'kludges' in the popular press and even some academic literatures. A commonly cited example of a kludge is the bony flatfish (e.g., Dawkins, 1986, 1996). The ancestors of the flatfish used to swim vertically in the ocean. At some point in their 


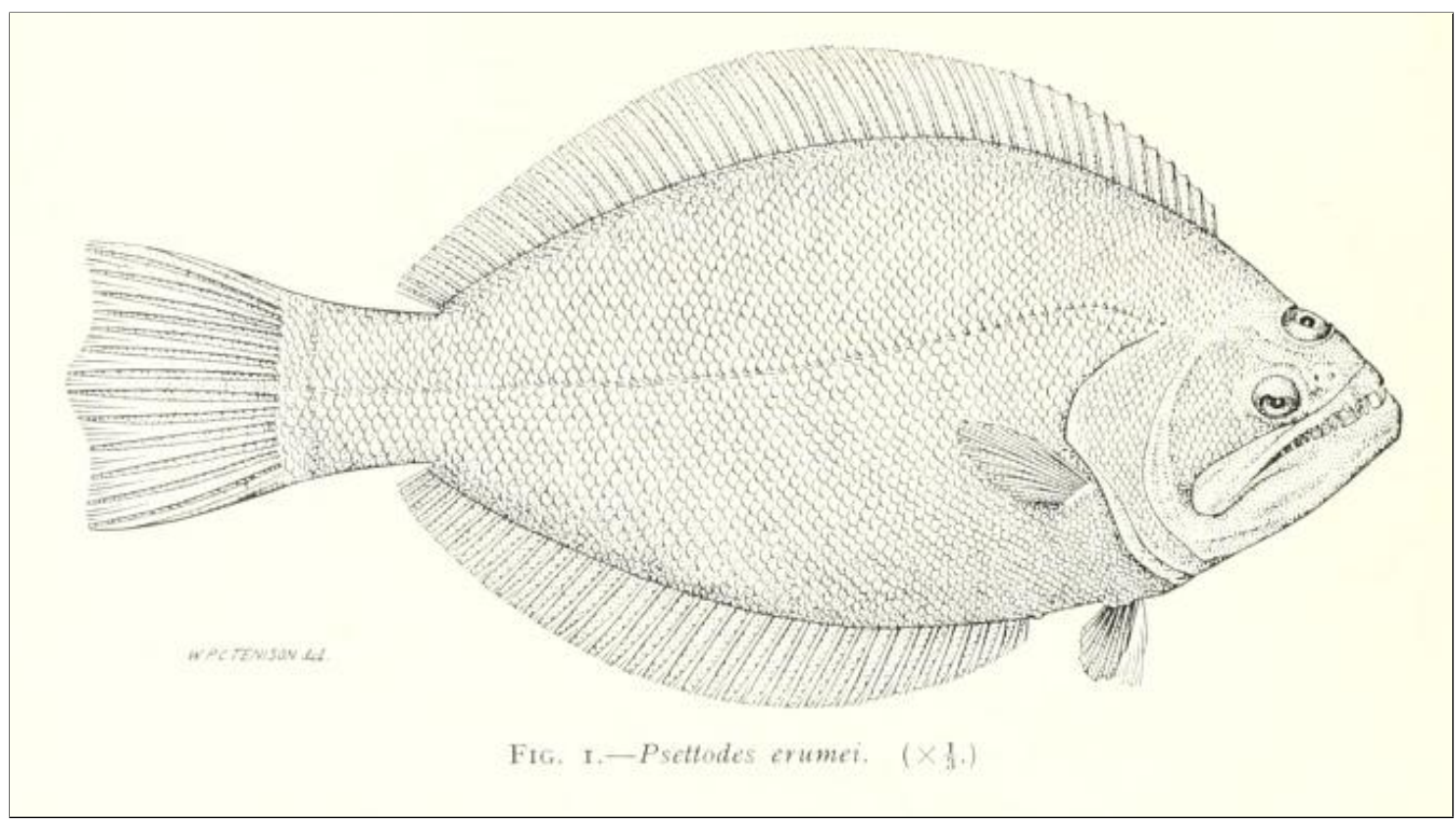

Figure 8: An example of a kludge: the bony flatfish. Source: Figure 1 of Norman (1934).

evolutionary history, the fish began living on the ocean floor, lying on one side, leaving one eye looking directly at the sand. Over time and through evolutionary processes, the eye migrated to the top of the head so that the two eyes were on one side of the fish and looking up (Friedman, 2008). An example of the modern flatfish is shown in Figure 8, which is the first drawing in Norman's (1934) collection of flatfish sketches. This incremental innovation solved an inefficiency given the situation at the time but resulted in an overall design that was suboptimal. If one were to completely redesign the bony flatfish, one would have the fish lie on its stomach so that neither eye would be in the ground and symmetry could be maintained. The more the flatfish evolved to its current form, the further away from this ideal it became.

Although the insight this provides for the social sciences is still under-explored, there are a few studies within economics that attempt to make progress in this direction. One is a theoretical analysis by Ely (2011), who provides a formal framework to understand kludges, which he defines as "an improvement upon a highly complex system that solves an inefficiency but in a piecemeal fashion and without addressing the deep-rooted underlying problem ... the kludge itself - because it makes sense only in the presence of the disease it is there to treat - intensifies the internal inefficiency, necessitating either further kludges in the future or else eventually a complete revolution." (p. 211)

A subsequent paper takes a more applied approach and uses the notion of kludges to better 
understand the evolution of laws or policies (Kawai, Lang and Li, 2018). The paper models the emergence of kludges in policy in a world with two political parties. In this setting, when a new political party comes into power they can add or remove previous rules in an incremental manner. An interesting aspect of the model is that rules can be deleted. That is, it is possible to move 'downhill'. However, the model shows how despite this ability, the political parties choose not to move 'downhill' removing old rules, but instead only choose to add new rules. This occurs because as policies become more complex - i.e., with a longer path of rules being added on the rules become entangled in one another. And so removing an unfavorable rule involves also removing favorable rules if they are entangled. Thus, when policy complexity is high, there is entanglement and a bias towards adding rather than deleting rules, generating further additions and complexity.

The model shows that even in settings that, ex ante, are different from that of biological evolution where one can undo innovations, in equilibrium, the environment mirrors biological evolution and kludges emerge. Interestingly, the theory shows that policy is more likely to exhibit kludges if political power is balanced, electoral terms are short, and legislative frictions are high. These are all characteristics of the U.S. political system. The model also predicts that with a kludged public policy, the policy outcomes are moderate, which is also consistent with the reality of the United States.

There are many examples of cultural evolution that can be described as kludges. Consider the issue of paternity certainty. While men can create multiple children during a short period of time, women have biological limits on how quickly they can have children. Thus, the main concern for women is to ensure that the father of her children invests in them sufficiently. That is, she does not want him to employ a strategy where he is choosing quantity over quality. The concern of a potential father is that he may invest heavily in a child thinking that it is his but it may not be. For biological reasons, while a woman is certain that a child is her offspring, there is much less certainty for men. If there is sufficient paternity uncertainty, then he will be less likely to invest in his mate's child.

This is a fundamental issue related to reproductive success which is driven by the nature of reproduction and the biological differences between men and women. The existing evidence indicates that societies came up with different innovations to improve upon this. Much like Dawkins' (1996) analogy of reaching peaks by climbing uphill one step at a time, different societies 
climbed different hills; customs and traditions evolved that served to improve upon this issue.

Among certain groups, matrilineal kinship developed. Here kinship is traced through the women. Men belong to the lineage of their sisters and mother and children belong to the lineage of their mother. This means that a father does not belong to the same lineage as his children. The children belong to their mother's lineage and their father belongs to his mother and sister's lineage. Because of this, the father is not the most important male adult in a child's life and he is not the one providing primary financial support. The people who fill this role are the mother's brothers (i.e., the child's maternal uncles). The logic behind this is that with paternity uncertainty, there is no guarantee that the father is genetically related to a child. However, it is certain that the mother's brother is related to the child. The child came from their mother and so is related to her. The mother and her brother came from the same mother and so they are related. Thus, the child must be related to their uncle. There is no guarantee that the child is related to the father.

Thus, incremental innovation in the face of paternity certainty is matrilineal kinship, where family/clan membership is traced through women. The results of such an incremental innovation are far reaching and and can be difficult to undo. In this case, it is natural that family members live in close proximity to one another, and, thus, there is matrilocality, where the nuclear family lives near the woman's family.

An alternative incrementally-designed set of innovations that has emerged is as follows. Rather than having matrilineal kinship, a society has patrilineal kinship. Lineage is traced through the father's line. At the time of marriage, the wife leaves her lineage and joins her husband's. Due to the tendency of lineages to live within close proximity, patrilocality develops where families live close to the husband's parents.

This then presents another problem. If daughters leave their parents lineage and move away from them when they are married, what incentive is there for them to invest resources in raising someone who they will never see again and no longer be part of their family after marriage? The incremental innovation that emerged to overcome this issue is bridewealth, where a sizeable transfer of resources from the husband's family to the wife's family at marriage. This is given as a gift of appreciation and incentivizes the investment of resources in a daughter even knowing that she will not be a member of the family in the future. The practice of bridewealth is infrequently observed in matrilineal societies.

The two evolved solutions to the problem of paternity uncertainty and paternal investment 
in children are very different and take societies along different paths with different incremental improvements. Presumably, one equilibrium is better than the other. For example, Lowes (2018) finds that within matrilineal households, due to the greater empowerment of women, children are healthier and more educated. Thus, it is plausible that this evolutionary path is better for fitness. However, once a society has progressed down the patrilineal path, jumping to the matrilineal equilibrium is no easy task. This would involve undoing existing customs, leading to a lower payoff - what Dawkins (1996) calls 'moving downhill' - to then be able to travel along the matrilineal path. One would need to undo bridewealth, patrilocality, and fundamentally alter a society's perception of kinship.

This is potentially an example of a kludge. As the cultural innovations accumulate, the society is locked in more deeply into a suboptimal equilibrium. The case for a kludge among patrilineal societies is particularly strong for those who have developed additional cultural innovations that are harmful to women but are meant to resolve the issue of paternity certainty. As has been documented, in societies that practice nomadic pastoralism, where men are often absent for long periods of time, the problem of paternity certainty is particularly acute (Becker, 2019). In these societies, it appears as if additional innovations are required. It is not possible to move 'downhill' and then back 'uphill' to the higher matrilineal 'peak'. Instead one must continue upward on the existing path.

As documented in Becker (2019), female genital cutting and infibulation developed as customs that helped husbands ensure greater paternity certainty. With these extreme forms of female circumcision, sex becomes painful, reducing the temptation to have sexual relations outside of the marriage, even when the husband is away for extended periods of time. In other parts of the world, other customs emerged that also served the purpose of controlling women's autonomy and sexuality, such as the veil or burqa, restrictions on women's mobility, and limits on the decisions that women could make.

\section{Group-Level Selection}

Our discussion up to this point has implicitly assumed that the success of a cultural trait depends on the wellbeing of the individuals with the trait. However, historically a big part of cultural evolution occurs through the rise and fall of societies. In other words, the fate of individuals and their success is as much tied to the success of their community as their own relative success 
within the community. Thus, what may also be important for the adoption of cultural traits is how they affect the society as a whole. This is called group-level selection and is often used as one explanation for why prosocial cultural traits (ones that are costly for the individual but beneficial for the group), like altruism or proclivity to cooperate, can emerge (Henrich, 2004a, Richerson, Baldini, Bell, Demps, Frost, Hillis, Mathew, Newton, Naar, Newson, Ross, Smaldino, Waring and Zefferman, 2016).

This insight has the potential to increase our understanding of the history process, particularly at the society level. To see this, recall the famous quote from Charles Tilly (1990) that "war made the state, and the state made war" (p. 42). While generally not viewed in this light, Tilly's famous argument that war lies at the heart of the economic rise of Europe is actually an argument about group-level selection. Those groups, namely states, with characteristics that were beneficial were able to outcompete other states with less beneficial characteristics. He writes that "states having access to a combination of large rural populations, capitalists, and relatively commercialized economies won out... their form of state became the predominant one in Europe. Eventually European states converged on that form: the nation states." (p. 15).

Recent studies have examined the effects of conflicts, particularly within the European context (Gennaioli and Voth, 2015, Dincecco and Onorato, 2017). Studies have explored the effects that warfare had on taxation, urbanization, and state formation. However, the most obvious mechanism outside of economics, group-level selection, has not been explicitly considered, even though the famous effects of Tilly can be interpreted through this lens. ${ }^{15}$ With the formation of groups, such as villages, kingdoms, empires, or nation-states, the wellbeing of the citizens of each entity are intricately connected. If a kingdom is conquered, everyone in the Kingdom is conquered. Thus, warfare and interstate conflict are a form of group-level competition. This is important because this, in turn, leads to group-level selection. ${ }^{16}$ When an individual's success is, to a large extent, determined by the success of the group rather than their own success, then cultural traits that are not individually optimal but are socially beneficial can arise. An example of such a trait is altruism. Giving to others when they are in need reduces our material wellbeing.

\footnotetext{
${ }^{15}$ The most extensive research undertaken in this direction is from the evolutionary biologist Peter Turchin, who applies insights from evolutionary biology to understand the course of human history, including warfare and the rise and fall of empires. For a sampling of this ambitious research agenda, see Turchin $(2003,2006,2016)$ and Turchin, Currie, Turner and Gavrilets (2013).

${ }^{16}$ To be precise, there are two conditions that are needed for warfare to cause cultural group selection. The first is that variation in cultural traits between groups causes success in warfare. The second is that success in warfare affects the spread of the cultural traits (Richerson et al., 2016).
} 
However, if everyone in a society does this, then the society will be better functioning and will be better off. Of course, just because traits are socially beneficial does not mean they will arise in equilibrium. This depends on the trade-offs between group-level benefits and individual-level costs. The stronger group-level selection is the more likely it is that the former will prevail over the latter (Henrich, 2004a).

The prediction that arises from the framework of group-level selection is that conflict should be associated with more prosocial traits (i.e., traits that are socially beneficial). Several recent studies have stumbled across the counter-intuitive finding that conflict seems to be associated with higher levels of prosociality and social cohesion. This was confirmed in a recent metaanalysis Bauer, Blattman, Chytilova, Henrich, Miguel and Mitts (2016). The finding is odd if one expects that conflict would cause the breakdown of social capital. However, viewed through the lens of intergroup competition, these relationships make sense. Evolutionary theory predicts an association between conflict and prosocial behavior. Such an effect may be more pronounced during periods of conflict. That is, conflict may activate prosocial psychology which is particularly beneficial in that setting.

Another explanation for the relationship comes from very recent research in social psychology that emphasizes the effect that 'threat' has on the the extent to which norms are adhered to - i.e., their 'tightness.' According to Gelfand (2018), external threat increases the importance of norm following and therefore tightness increases. If conflict is a form of threat and a society's baseline norms are ones of cooperation and prosociality towards in-group members, then past experience with conflict will lead to greater prosociality. Consistent with this interpretation, subsequent research by Henrich, Bauer, Cassar, Chytilova and Purzycki (2019) shows that past experience with conflict is associated with greater religiosity, which can be interpreted as a tightening of traditional norms. The study uses a survey of 1,709 individuals from three post-conflict societies: Uganda, Sierra Leone and Tajikistan. They find that conflict exposure is associated with an increased likelihood of identifying as Christian or Muslim and with more frequent participation in religious gatherings and rituals.

A case study illustrating the logic of group-level competition is provided by Nunn and Sanchez de la Sierra (2017). The authors described events in a village in the Eastern Democratic Republic of the Congo. The village had a history of being regularly pillaged by a Hutu militia group called the FDLR. The raiding and pillaging had occurred multiple times per year for well over a decade. This 
was until 2012 when an elderly man in the village, through a dream, learned how to bulletproof the people of the village by having them undertake a specific ritual. Beliefs in bulletproofing are common in the region and are connected to traditional religious beliefs. As is common, this form of bulletproofing came with conditions that had to be followed for the protection to work. Thus, if someone who had been known to have been bulletproofed died, then it must be the case that they did not follow the necessary conditions. Due to the bulletproofing, the villagers, particularly the young men, were able to fight back when they were attacked. Over the course of multiple years, the FLDR eventually gave up trying to attack the village and concentrated their efforts elsewhere. For the first time in over a decade, the village had been liberated.

In their analysis, Nunn and Sanchez de la Sierra (2017) try to understand these events. They interpret the production function of producing the village's freedom as one with team production, meaning there are strong complementarities to effort. As is well known, the equilibrium level of effort is lower than is socially optimal in such settings. Beliefs in bulletproofing serve to reduce the perceived cost of effort and to raise the equilibrium level of effort so that it is closer to the socially optimal level. Thus, these beliefs, even if false, are beneficial for society. However, this benefit does not guarantee the existence of such beliefs. As discussed above, socially beneficial beliefs or preferences that are individually suboptimal only arise with strong group-level selection. The authors argue that the Eastern DRC which has experienced decades of sustained conflict is a setting where group-level selection is expected to be strong. Thus, in their setting, conflict generated group-level competition, which resulted in beliefs that are socially beneficial although individually suboptimal. This interpretation is consistent with the fact that beliefs about bulletproofing appear to be common in many conflict-ridden areas, not only in the contemporary period but also historically (Kamarck, 2009, ch. 4, Sinclair-Thomas and Challis, 2017).

The best evidence for whether historical warfare would have been significant enough to generate group-level selection is from a recent study by Samuel Bowles (2009). In it, he takes a more historical perspective and asks whether the group-level selective pressures due to intergroup conflict during Late Pleistocene and early Holocene could have been sizeable enough to affect the evolution of prosocial cultural traits. The study combines models of group-level selection, archaeological data from the period, and ethnographic data from contemporary hunter-gatherers. It finds that the levels of mortality associated with intergroup warfare would have been sizeable enough to have a large effect on prosocial traits, promoting behaviors that while individually 
costly would have been beneficial for the group overall.

While the examples discussed to this point have focused on warfare as the source of group-level competition, the exact same logic applies to competition that occurs through market forces. While this form of competition might not be particularly important for those studying cultural evolution over the course of human history, it is highly relevant for the field of economics where markets are central.

One of the few pieces of research along these lines is a recent study by Francois, Fujiwara and van Ypersele (2018). In the study, the authors examine selection arising from firm competition. They begin by first showing a relationship between the competitiveness of an industry and the level of trust of individuals who work in that industry. They look at both the United States and Germany and show this relationship holds both in the cross-section and over time for workers who switch industries. In this setting, firms are the group and firm competition is the form of group-level competition. Given this, group-level selection is expected to facilitate the emergence of group-level beneficial traits, like generalized trust. Firms with trusting employees perform better and are more likely to survive. This is true despite evidence that suggests that if trust is too high it can lower individual income (Butler et al., 2016). In other words, even though trust is socially beneficial it is not necessarily individually beneficial.

The authors confirm these findings and their interpretation by undertaking an event study exploiting the wave of bank deregulation across U.S. states in the 1980s, which led to more competitive industries. Lastly, they show that the effect of group-level selection on generalized trust can be replicated in a behavioral experiment. Using 220 participants from France, individuals played a series of one-shot anonymous public goods games against others within a group of 20 individuals. One of two randomly-selected groups had payoffs that were determined by the relative performance of their group compared to another. They find that group-level selection, resulted in higher contributions, and importantly in higher levels of self-reported trust at the end of the experiments.

The study is important because it provides valuable evidence that market competition can result in group-level selection. This provides a new perspective from which one can think about market competition and its effects. 


\section{E. Biology, Sex, and Gender}

There are well-known biological differences between men and women among the human species. Relative to women, men tend to be taller, larger in stature, and have greater upper body strength (Ruff, 2002, Gustafsson and Lindenfors, 2004). The two sexes have very different roles in reproduction. The role of men is to fertilize an egg, while that of women is to carry the egg as it develops, give birth, and (typically) breastfeed the newborn for the first years of life.

These biological differences place different constraints on reproduction for the two sexes. Women can only have only one offspring (ignoring the case of twins) per 1-2 year period. By contrast, men can have a much larger number of offspring during this same time. However, due to the nature of sex and childbirth, men have much less certainty that a child that is born is theirs biologically. By contrast, women have essentially perfect certainty that a child is biologically her offspring.

This suggests different biological concerns for men and women and as a consequence different optimal mating strategies, which have been studied extensively (e.g., Trivers, 1972, Buss and Scmitt, 1993, Buss, 1994). As we will see, these strategies have implications for gender relations and social structure more generally. For women, the concern is that men will choose a quantity over quality mating strategy and not invest sufficient resources into the child. For men, the concern is that the child is not theirs and they are spending resources on another male's child. If this is believed, they may not invest the necessary resources to raise the child.

These biologically-based concerns have important social consequences that have been studied in the economic history literature. As mentioned above, Becker (2019) studies how the fundamental issue of paternity uncertainty differs depending on ecological conditions and what effects this has on the social structure in society. Her study shows that parts of the world that are particularly well-suited to pastoralism - namely, places that are marginal for agriculture but suitable for the grazing of animals - exhibit much greater control of woman's sexuality by men. This can take the form of norms about female decision-making autonomy and practices such as infibulation and veiling. This is because, in a pastoral society, grazing requires men to be absent from the community as their herds move to new pastures. These prolonged periods of absence raised concerns of infidelity, which resulted in the evolution of restrictions on female sexuality.

Another evolved response to the issue of paternal uncertainty has been matrilineal kinship. Rather than the father being the primary supporter of a child, it is the mother's brother (i.e., 
the maternal uncle). With matrilineal kinship, lineage is traced through women. Men belong to the lineage of their sisters and mothers, and children belong to the lineage of their mother. Thus, a father does not belong to the same lineage as his children. The children belong to their mother's lineage and the father belongs to his mother and sister's lineage. As a consequence, the father is not the most important male adult in his children's lives and, often, he is not the one who provides primary financial support. Instead, this is the mother's brothers (i.e., the children's maternal uncles). As explained above, the logic behind this is that while there is no guarantee that the father is genetically related to the children, it is certain that the mother's brother is related to the children. Thus, the fundamental problem of how to incentivize the father to invest in his children if perceived paternal uncertainty is high is solved by having developed norms and customs where maternal uncles are the ones who invest in children.

As we have discussed, matrilineal kinship and control over women's sexuality are two different evolutionary paths that serve to address the same fundamental biologically-based problem. A testable prediction of this evolutionary perspective behind these customs is that the two customs are substitutes and should be negatively correlated in the ethnographic record. Consistent with this, one does find that matrilineal kinship is associated with greater female empowerment (Lowes, 2018). In addition, if one looks at ethnographic data, one finds that matrilineal kinship is associated with weaker control of women's sexuality. Although data are limited, there is some information on these cultural traits in the Ethnographic Atlas. Within the database, there are 244 patrilineal and 59 matrilineal ethnic groups for which data are available on societal norms about premarital sexual behavior of girls, which we take to be an indicator of sexual norms more generally. For matrilineal groups with data, only $6.7 \%$ (4 of 59) have norms that prohibit any sex before marriage. Among patrilineal groups with data, the same statistic is $34 \%$ (82 of 244). By contrast, among matrilineal groups with data, 54\% (32 of 59) have norms that fully permit premarital sex, while among patrilineal groups with data, this figure is only $33 \%$ (80 of 244 ).

Another biological difference between men and women is differences in their body size and strength, which is an example of a more general biological phenomenon referred to as sexual dimorphism. Differences between men and women has been fairly stable (i.e., a $15 \%$ difference in body mass) for Homo sapiens since the Pleistocene (Ruff, 2002). Recent research within economics has studied the consequence of sexual dimorphism for modern gender norms and how it is mediated by historical technologies that made the physical differences more or less important. 
Alesina, Giuliano and Nunn (2013b) provide evidence that the greater physical size and strength of males was an important determinant of a gender-based division of labor historically, but primarily in agricultural societies that had adopted intensive plow agriculture. With this form of agriculture, significant upper body strength was needed to control or pull the plow. As a consequence, in these societies, there was a gendered division of labor where men worked outside the home in agriculture and women tended to work within the home. As shown by Alesina et al. $(2013 b)$, this division generated norms about the appropriate role of women in society, which persist in the modern era and affect female employment outside the home today. The form of traditional agriculture has even been found to affect preferences for sons over daughters, which results in male-biased sex-ratios (Alesina, Giuliano and Nunn, 2018).

\section{Contributions of Economics to Cultural Evolution}

The study of cultural evolution is a recent area of research, with the bulk of findings emerging in the last few decades. The field began with a small number of scholars who adapted models from evolutionary biology to cultural evolution (e.g., Cavalli-Sforza and Feldman, 1981, Boyd and Richerson, 1985). An important contribution of economics has been to use these theoretical foundations to build more elaborate models that remain tractable and provide insight into a range of economic issues, including preference heterogeneity and segregation (Bisin and Verdier, 2000, 2001b), entrepreneurship (Doepke and Zilibotti, 2014), occupational choice (Doepke and Zilibotti, 2008), female labor force participation (Fernandez, 2013), parenting strategies (Bisin and Verdier, 2001a, Doepke and Zilibotti, 2017), or domestic institutions (Tabellini, 2008, Bisin and Verdier, 2017). A related area of research that has developed within economics is theoretical work that exploits the dynamics of natural selection to understand various aspect of economic growth over the very long-run (e.g., Galor and Moav, 2002, Galor and Michalopoulos, 2012, Galor and Ozak, 2016, Galor and Savitskiy, 2018).

One way to think about the difference between the theoretical analysis of cultural evolution within evolutionary anthropology and economics is that the prior has tended to focus on studying the emergence of culture and its transmission between individuals. Thus, culture and its evolution is the outcome of interest in the models. By contrast, within economics, culture and 
its transmission is taken as given and the analysis follows based on this assumption. ${ }^{17}$ Thus, the theoretical work within evolutionary anthropology provides the micro foundations for the assumptions used in a wide range of models within cultural economics.

While the origins of the field of cultural evolution were primarily theoretical, the field has subsequently become increasingly quantitative (Mesoudi, 2016). It is this line of analysis to which economic historians and growth economists have made the greatest contributions. A large body of evidence documents how the historical environment or historical shocks - what other disciplines would call a society's ecology - affects the long-term evolution of cultural traits. ${ }^{18}$ For the interested reader, an overview of this body of evidence is provided in Table 1 . The table reports the citation, the cultural trait that is being explained, the determinant which is the focus of the paper, the unit of observation, the sample, and the primary statistical method used. Although the list provided is certainly incomplete, it still illustrates that economists have made important contributions to a deeper understanding of the historical and ecological factors that have shaped the evolution of cultural traits.

Very recently, there has been a push for multidisciplinary research that takes a historical/evolutionary perspective, involving both economists and evolutionary anthropologists. An example is a recent collaboration between anthropologist Joseph Henrich and three economists (Schulz, Bahrami-Rad, Beauchamp and Henrich, 2019). Together, the authors study the historical origins of Western European psychology. They document how the policies of the medieval Roman Catholic Church, such as the prohibition on cousin marriage, weakened extended kinship ties and lead to a more individualistic social structure and psychology. To do this, the authors used data from a range of disciplines, including historical data on the duration of exposure to the medieval Western church, ethnographic data on the traditional prevalence of kinship practices like cousin marriage and polygamy, and contemporary data on various psychological and behavioral characteristics.

Another way that economics has contributed to the study of cultural evolution is by evidence for the effects that shorter-term factors can have on cultural traits. This literature is summarized in Table 2. It has the same structure as Table 1 except that it summarizes studies that estimate the more-immediate effects of the factors of interest; namely, effects that are felt within a person's

\footnotetext{
${ }^{17}$ An exception is Bisin and Verdier (2001a).

${ }^{18}$ See Nunn (2021) for a summary of this literature.
} 


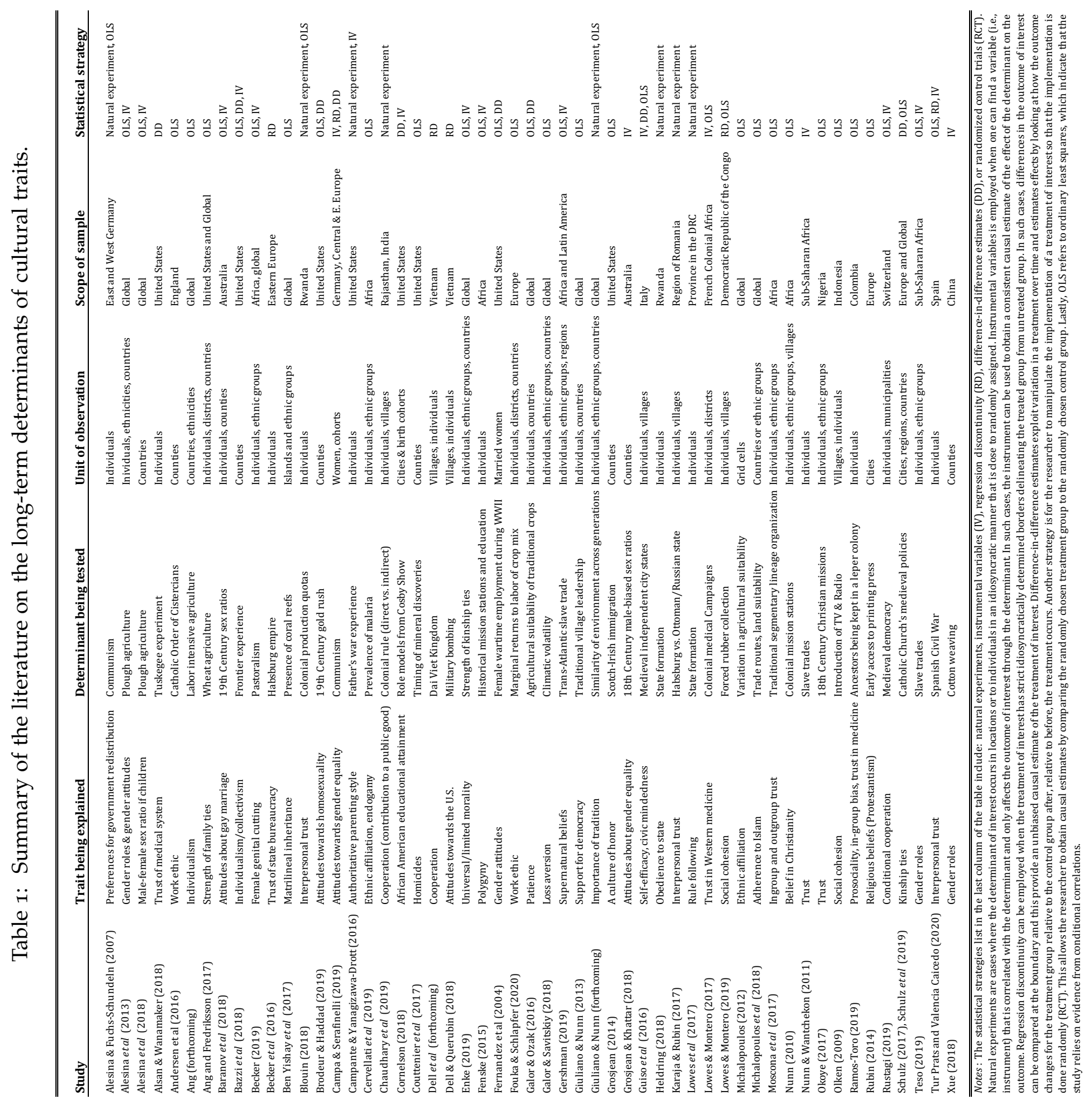




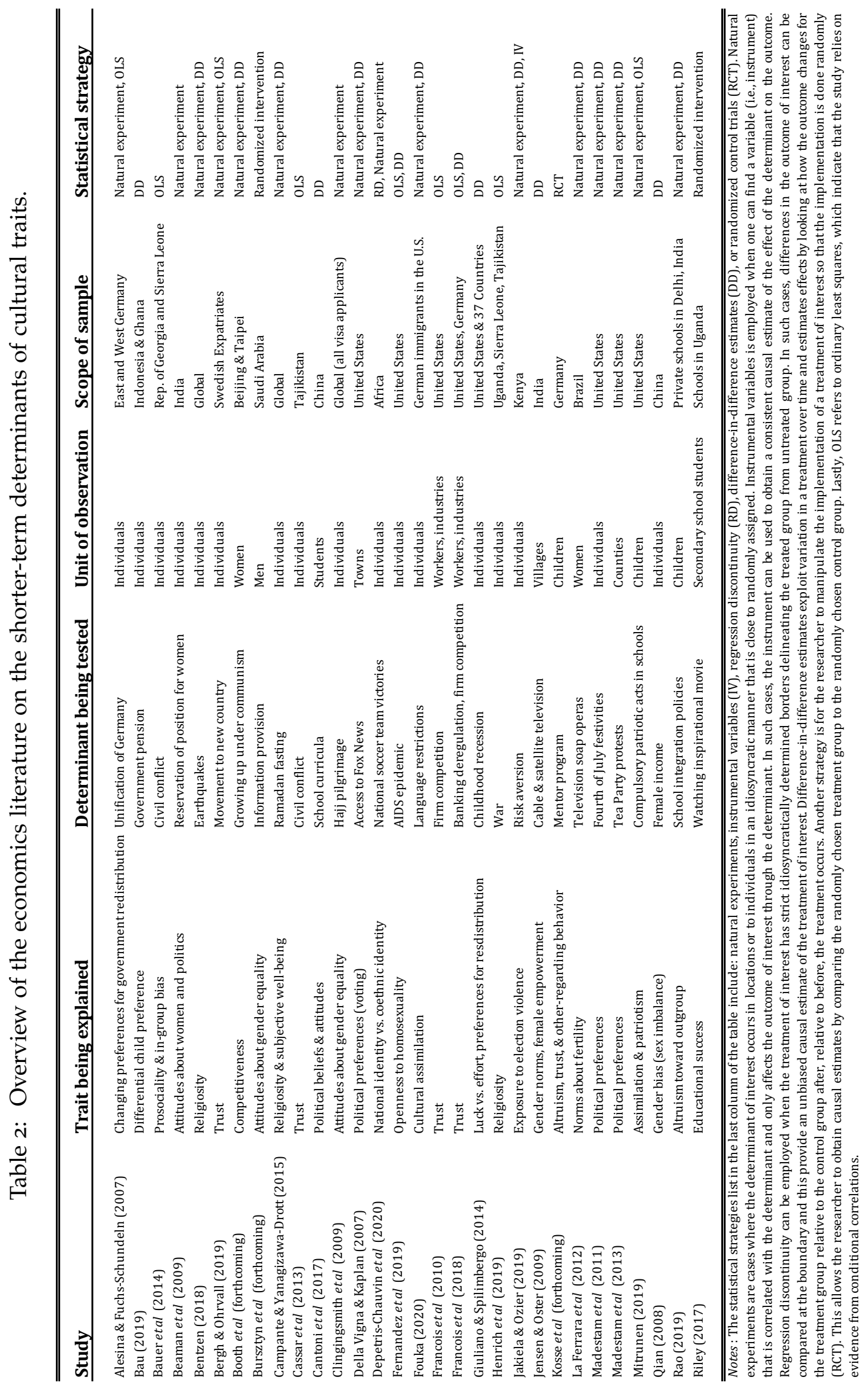




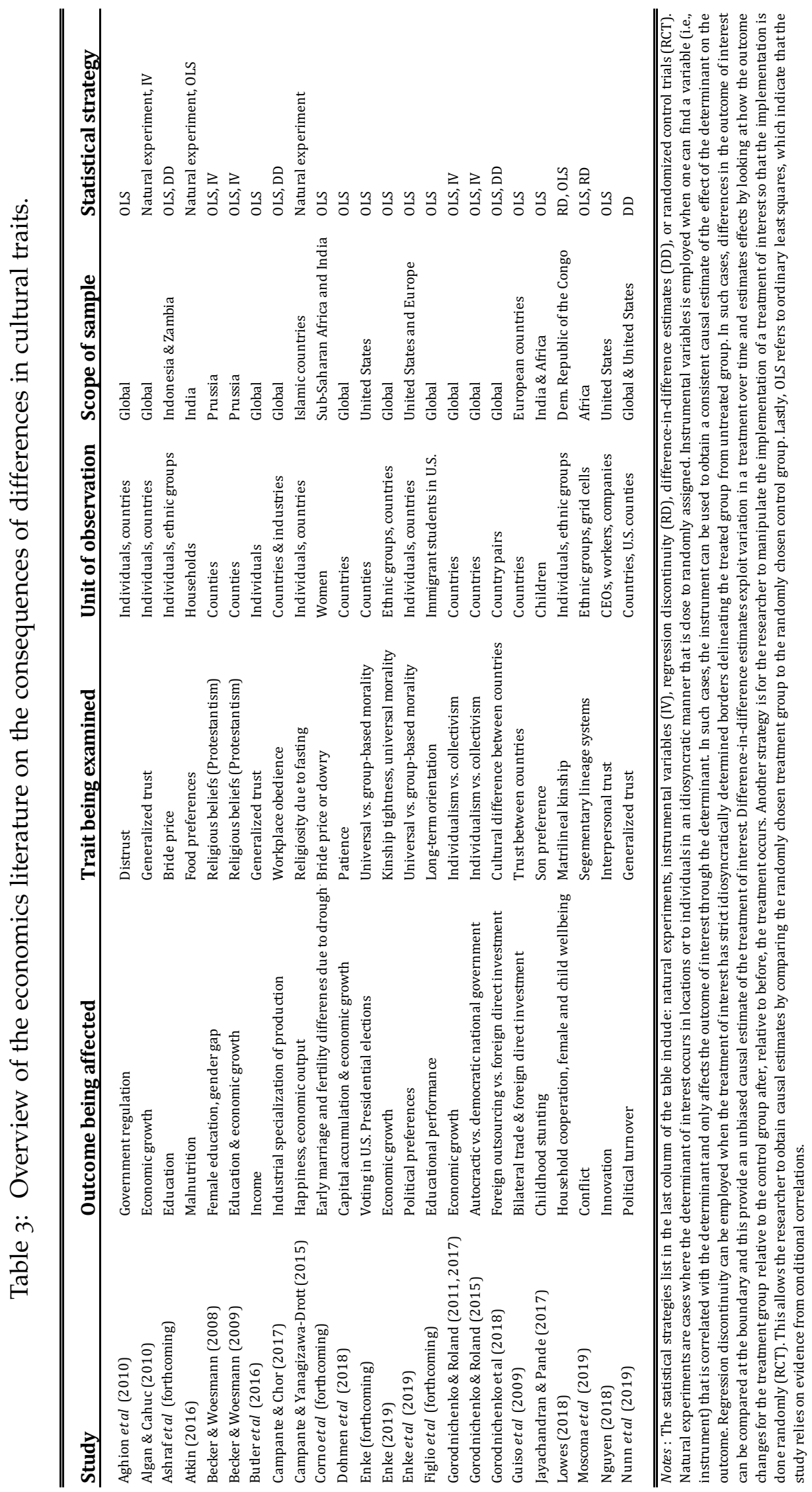


lifetime rather than multiple generations into the future.

An additional contribution that economics has made is to estimate the effects that cultural traits have on economic outcomes. Research has shown that culture affects a host of outcomes, including trade, foreign direct investment, political turnover, conflict, innovation, savings, investment, and economic growth. An overview of the key studies from this body of research is provided in Table $3 \cdot{ }^{19}$

A particularly noteworthy aspect of the body of economic research summarized in Tables 1-3 is that the scope of societies studied is relatively broad. This can be seen with a quick glance at the 'Scope of sample' column in the three tables. There are many studies of non-Western societies. This is important since it is now well-recognized that the cultural traits of Western European societies - what Henrich, Heine and Norenzayan (2010b) famously term 'WEIRD' societies - are not the modal or even median traits of the World. Instead, they appear to be highly exceptional (Henrich et al., 2010b, Henrich, 2020, Muthukrishna, Bell, Henrich, Curtin, Gedranovich, McInerney and Thue, 2020). Many disciplines that seek to better understand human culture and human behavior, most notably psychology, have a very strong tendency to focus almost exclusively on Western societies (Henrich, Heine and Norenzayan, 2010a). In addition, because they are not particularly interested in cross-cultural variation, they then do not ask the question of where this variation is from, which would then cause them to look at history for answers. Thus, there is also a tendency for analysis to be ahistorical (Muthukrishna and Slingerland, forthcoming). While behavioral economics often shares the same 'WEIRD' bias, research within cultural economics generally considers a broad cross-section of societies and takes a historical perspective. The reason for this approach is likely due to the origins of the field, which lie in economic history, economic development, and economic growth, each of which has traditionally been historical and/or global in their focus. In the future, expect research in this area to continue to become increasingly interdisciplinary, particularly as fields like psychology begin to look beyond WEIRD societies and then seek to understand the historical origins of cross-societal differences on culture and psychology.

An important contribution that I can envision economists - particularly economic historians and political economists - making to evolutionary research is a greater emphasis on the presence of power and coercion and an explicit inclusion of states and state actors into theory and empirics.

\footnotetext{
${ }^{19}$ For a review of the early literature documenting the economic consequences of culture see Guiso et al. (2006).
} 
In the simplest evolutionary models, the evolution of particular traits depends on the relative success of each. However, in reality, the success of different groups often depends on the institutions and power structures within societies. While there are existing evolutionary studies that incorporate power, coercion, and institutions (e.g., Vehrencamp, 1983), there is scope for a greater and more explicit focus of these aspects of human society within evolutionary frameworks. While there has already been important progress using an evolutionary perspective to think about states and institutions (e.g., Bowles and Gintis, 1998b,a, Gintis, 2000, Platteau, 2000, Aoki, 2001, Bowles and Gintis, 2002, Bowles, 2004, Tabellini, 2008, Bisin and Verdier, 2017, Besley and Persson, forthcoming), there is much more that remains to be done.

A recent example of research along these lines is the theoretical study by Tabellini (2008) which models the interplay between culture and institutions. In the model, a cooperative cultural trait evolves through vertical transmission. He then introduces institutions, which are 'rules of the game' that affect the payoffs to the different cultural types in the model. The institutions are determined by majority voting. A potential equilibrium in the model is one where there are a large number of non-cooperative types. They vote for institutions that do not punish those who do not cooperate, which in turn increases the payoffs to non-cooperators. In another equilibrium, the number of cooperators is high, they are able to implement institutions that punish deviations from cooperation, which reduce the payoff to non-cooperators and increase the payoffs to cooperators, thus sustaining this equilibrium. The model, therefore, shows how culture, which is endogenous to the institutional structure, underpins the type of institutions that emerge.

While I expect research on the interplay between culture and institutions to develop further - see Bisin and Verdier (2017) for a more recent example - I also expect the empirical research on these questions to also develop further. There are a few examples of recent papers that have sought to understand the effect of states and institutions on the evolution of cultural traits, but much more remains to be understood. For example, the effect of state presence appears to be different in the limited number of settings that have been studied and we really don't have a clear sense of why yet (e.g., Becker, Boeckh, Hainz and Woessmann, 2016, Lowes, Nunn, Robinson and Weigel, 2017, Dell, Lane and Querubin, 2018, Heldring, 2020).

More generally, whatever specific direction future research takes, I expect that the contribution of economists, particularly economic historians and political economists, to the study of cultural evolution will continue to increase. 


\section{Conclusions}

In this chapter, I considered the benefits of viewing history through an evolutionary lens. The first part of the chapter focused on thinking about the benefits of culture, discussing both the theory and empirical evidence for the benefits of cultural evolution. The primary benefit of culture is that it allows one to conserve on information acquisition costs and to relying on the accumulated body of knowledge that has evolved during previous generations or in other parts of society. Thus, culture allows the collective knowledge of a society to be much greater than the information that any one person could learn in their lifetime. The theoretical models and empirical evidence that I reviewed convincingly show that societies are better off when decision making occurs through cultural processes.

I then turned to a discussion of how an evolutionary perspective provides insights into a range of phenomena that are important within economics. I discussed how insights into the reality of knowledge and innovation can be gained by thinking of these as cumulative cultural processes. I discussed how an understanding the nature of cultural evolution helps us better understand the nature of historical persistence, path dependence, and variation in the success of societies. I discussed evolutionary insights that improve our understanding of human history. These included concepts such as environmental mismatch, the collective brain, cumulative improvements, kludges, group-level selection, sexual dimorphism, and reproduction strategies. I discussed how these provide insights into key aspects of economics history; namely, including human capital, innovation, warfare, state formation, cooperation, social structure, gender roles, kinship, social structure, path dependence, and comparative economic development.

I ended the chapter by discussing the recent wave of research within economic history and growth economics that studies, both theoretically and empirically, cultural evolution and its importance for social and economic outcomes. I discussed the way this research has contributed to the field of cultural evolution and how I expect this to continue in the future.

\section{References}

Aghion, Philippe, Yann Algan, Pierre Cahuc, and Andrei Shleifer, "Regulation and Distrust," Quarterly Journal of Economics, 2010, 125 (3), 1015-1049.

Alesina, Alberto and Nicola Fuchs-Schundeln, "Good-Bye Lenin (or Not?): The Effect of Communism on People's Preferences," American Economic Review, 2007, 94 (4), 1507-1528. 
_ , Edoardo Teso, and Stefanie Stantcheva, "Mobility and Preferences for Redistribution," American Economic Review, 2013, 108 (2), 521-554.

-, Paola Giuliano, and Nathan Nunn, "On the Origins of Gender Roles: Women and the Plough," Quarterly Journal of Economics, 2013, 128 (2), 469-530.

_, , , and _ , "Traditional Agricultural Practices and the Sex Ratio Today," PLoS ONE, 2018, 13 (1), e0190510.

Algan, Yann and Pierre Cahuc, "Inherited Trust and Growth," American Economic Review, 2010, $100(5), 2060-2092$.

_ and _, "Trust and Growth," Annual Review of Economics, 2013, 5, 521-549.

Alsan, Marcella and Marianne Wanamaker, "Tuskegee and the Health of Black Men," Quarterly Journal of Economics, 2018, 133 (1), 407-455.

Andersen, Thomas Barnebeck, Jeanet Bentzen, Carl-Johan Dalgaard, and Paul Sharp, "PreReformation Roots of the Protestant Ethic," Economic Journal, 2016, 127 (604), 1756-1793.

Andrews, Michael, "Bar Talk: Informal Social Interactions, Alcohol Prohibition, and Invention," 2019. Working paper.

Ang, James B. and Per G. Fredriksson, "Wheat Agriculture and Family Ties," European Economic Review, 2017, 100, 236-256.

_ and _ , "Wheat Agriculture and Family Ties," Journal of Economic Growth, forthcoming.

Aoki, Masahiko, Towards a Comparative Institutional Analysis, Cambridge: MIT Press, 2001.

Ashraf, Nava, Natalie Bau, Nathan Nunn, and Alessandra Voena, "Bride Price and Female Education," Journal of Political Economy, 2020, 128 (2), 591-641.

Ashraf, Quamrul and Oded Galor, "The "Out of Africa" Hypothesis, Human Genetic Diversity, and Comparative Economic Development," American Economic Review, 2012, p. forthcoming.

Atkin, David, "The Caloric Costs of Culture: Evidence from Indian Migrants," American Economic Review, 2016, 106 (4), 1144-1181.

Baranov, Victoria, Ralph De Haas, and Pauline Grosjean, "Men. Roots and Consequences of Masculinity Norms," 2018. Working paper, University of Melbourne.

Bau, Natalie, "Can Policy Change Culture? Government Pension Plans and Traditional Kinship Practices," 2019. Working paper, University of California Los Angeles.

Bauer, Michal, Alessandra Cassar, Julie Chytilova, and Joseph Henrich, "War's Enduring Effects on the Development of Egalitarian Motivations and In-Group Biases," Psychological Science, 2014, 25 (1), 47-57.

_ , Christopher Blattman, Julie Chytilova, Joseph Henrich, Edward Miguel, and Tamar Mitts, "Can War Foster Cooperation?," Journal of Economic Perspectives, 2016, 30 (3), 249-274.

Bazzi, Samuel, Martin Fiszbein, and Mesay Gebresilasse, "Frontier Culture: The Roots and Persistence of "Rugged Individualism" in the United States," 2018. Working paper, Boston University. 
Beaman, Lori, Raghabendra Chattopadhyay, Esther Duflo, Rohini Pande, and Petia Topalova, "Powerful Women: Does Exposure Reduce Bias?," Quarterly Journal of Economics, 2009, 124 (4), 1497-1540.

Becker, Anke, "On the Economic Origins of Restrictions on Women's Sexuality," 2019. Working Paper, Harvard University.

Becker, Sascha O. and Ludger Woessmann, "Luther and the Girls: Religious Denomination and the Female Education Gap in Nineteenth-Century Prussia," Scandinavian Journal of Economics, 2008, 110 (4), 777-805.

_ and _, "Was Weber Wrong? A Human Capital Theory of Protestant Economic History," Quarterly Journal of Economics, 2009, 124 (2), 531-596.

_ , Katrin Boeckh, Christa Hainz, and Ludger Woessmann, "The Empire is Dead, Long Live the Empire! Long-Run Persistence of Trust and Corruption in the Bureaucracy," Economic Journal, 2016, 126 (590), 40-74.

Bell, Alex, Raj Chetty, Xavier Jaravel, Neviana Petkova, and John Van Reenen, "Who Becomes an Inventor in America? The Importance of Exposure to Innovation," Quarterly Journal of Economics, 2019, 134 (2), 647-713.

Benjamin, Daniel J., David Cesarini, Christopher F. Chabris, Edward L. Glaeser, David I. Laibson, Vilmundur Guonason, Tamara B. Harris, Lenore J. Launer, Shaun Purcell, Albert Vernon Smith, Magnus Johannesson, Patrik K.E. Magnusson, Jonathan P. Beauchamp, Nicholas A. Christakis, Craig S. Atwood, Benjamin Hebert, Jeremy Freese, Robert M. Hauser, Taissa S. Hauser, Alexander Grankvista, Christina M. Hultman, and Paul Lichtenstein, "The Promises and Pitfalls of Genoeconomics," Annual Review of Economics, 2012, 4, 627-662.

Bentzen, Jeanet Sinding, "Acts of God? Religiosity and Natural Disasters Across Subnational World Districts," Economic Journal, 2019, 129 (622), 2295-2321.

BenYishay, Pauline Grosjean Ariel and Joe Vecci, "The Fish is the Friend of Matriliny: Reef Density and Matrilineal Inheritance," Journal of Development Economics, 2017, 127, 234-249.

Bergh, Andreas and Richard Ohrvall, "A Sticky Trait: Social Trust Among Swedish Expatriates in Countries with Varying Institutional Quality," 2019. Journal of Comparative Economics, forthcoming.

Besley, Timothy and Torsten Persson, "Democratic Values and Institutions," forthcoming. American Economic Review - Insights, forthcoming.

Billing, Jennifer and Paul W. Sherman, "Antimicrobial Functions of Spices: Why Some Like it Hot," Quarterly Review of Biology, 1998, 73 (1), 3-49.

Bisin, Alberto and Thierry Verdier, "Beyond the Melting Pot: Cultural Transmission, Marriage and the Evolution of Ethnic and Religious Traits," Quarterly Journal of Economics, 2000, 115, 955-988.

_ and _ , "Agents with Imperfect Empathy May Survive Natural Selection," Economics Letters, 2001, 71, 277-285.

_ and _ , "The Economics of Cultural Transmission and the Dynamics of Preferences," Journal of Economic Theory, 2001, 97, 298-319. 
_ and _, "On the Joint Evolution of Culture and Institutions," 2017. NBER Working Paper 23375 .

Bloom, Nicholas, Aprajit Mahajan, David McKenzie, and John Roberts, "Do Management Interventions Last? Evidence from India," American Economic Journal: Applied Economics, 2020, $12(2), 198-219$.

_, Benn Eifert, Aprajit Mahajan, David McKenzie, and John Roberts, "Does Management Matter? Evidence from India," Quarterly Journal of Economics, 2013, 128 (1), 1-51.

Blouin, Arthur, "Culture and Contracts: The Historical Legacy of Forced Labour," 2018. Working Paper, University of Toronto.

Booth, Alison, Elliott Fan, Xin Meng, and Dandan Zhang, "Gender Differences in Willingness to Compete: The Role of Culture and Institutions," Economic Journal, 2019, 129 (618), 734-764.

Bowles, Samuel, Microeconomics: Behavior, Institutions, and Evolution, Princeton: Princeton University Press, 2004.

-, "Did Warfare among Ancestral Hunter-Gatherers Affect the Evolution of Human Social Behaviors?," Science, 2009, 324, 1293-1298.

- and Herbert Gintis, "How Communities Govern: The Structural Basis of Prosocial Norms," in Avner Ben-Ner and Louis Putterman, eds., Economics, Values, and Organization, Cambridge: Cambridge University Press, 1998, pp. 206-230.

_ and _, "The Moral Economy of Communities: Structured Populations and the Evolution of Pro-Social Norms," Evolution and Human Behavior, 1998, 19, 3-25.

_ and _ , "Social Capital and Community Governance," Economic Journal, 2002, 112, F419-F436.

Boyd, Robert and Peter J. Richerson, Culture and the Evolutionary Process, London: University of Chicago Press, 1985.

_ and _ , "Why Does Culture Increase Human Adaptability?," Ethology and Sociobiology, 1995, $16,125-143$.

_ and _ , The Origin and Evolution of Cultures, London: Oxford University Press, 2005.

_, , , and Joseph Henrich, "The Cultural Evolution of Technology: Facts and Theories," in Peter J. Richerson and Morten Christiansen, eds., Cultural Evolution: Society, Technology, Language, and Religion, Strungmann Forum Reports, Cambridge, MA: MIT Press, 2013, pp. 119-142.

Brodeur, Abel and Joanne Haddad, "Institutions, Attitudes and LGBT: Evidence from the Gold Rush," 2018. Mimeo, University of Ottawa.

Burchardi, Konrad B., Thomas Chaney, and Tarek A. Hassan, "Migrants, Ancestors, and Investments," Review of Economic Studies, 2019, 86 (4), 1448-1486.

Bursztyn, Leonardo, Alessandra Gonzalez, and David Yanagizawa-Drott, "Misperceived Social Norms: Female Labor Force Participation in Saudi Arabia," forthcoming. American Economic Review.

Buss, David, The Evolution of Desire: Strategies of Human Mating, New York: Basic Books, 1994.

Buss, David M. and David P. Scmitt, "Sexual Strategies Theory: An Evolutionary Perspective on Human Mating," Psychological Review, 1993, 100, 204-232. 
Butler, Jeffrey V., Paola Giuliano, and Luigi Guiso, "The Right Amount of Trust," Journal of the European Economic Association, 2016, 14 (5), 1155-1180.

Campa, Pamela and Michel Serafinelli, "Politico-Economic Regimes and Attitudes: Female Workers under State-socialism," Review of Economics and Statistics, 2019, 101 (2), 233-248.

Campante, Felipe and David Yanagizawa-Drott, "Does Religion Affect Economic Growth and Happiness? Evidence from Ramadan," Quarterly Journal of Economics, 2015, 130 (2), 615-658.

_ and _ , "The Intergenerational Transmission of War," 2016. Working paper, Harvard Kennedy School.

_ and _, "Long-Range Growth: Economic Development in the Global Network of Air Links," Quarterly Journal of Economics, 2018, 133 (3), 1395-1458.

- and Davin Chor, “"Just Do Your Job”: Obedience, Routine Tasks, and the Pattern of Specialization," 2017. Working paper, Harvard Kennedy School.

Cantoni, Davide, Yuyu Chen, David Y. Yang, Noam Yuchtman, and Y. Jane Zhang, "Curriculum and Ideology," Journal of Political Economy, April 2017, 125 (2), 338-392.

Cassar, Alessandra, Andrew Healy, and Carl von Kessler, "Trust, Risk, and Time Preferences After a Natural Disaster: Experimental Evidence from Thailand," World Development, 2017, 94, 90-105.

_, Pauline Grosjean, and Sam Whitt, "Legacies of Violence: Trust and Market Development," Journal of Economic Growth, 2013, 18, 285-318.

Cavalli-Sforza, L.L. and M.W. Feldman, Cultural Transmission and Evolution: A Quantitative Approach, Princeton: Princeton University Press, 1981.

Cervellati, Matteo, Giorgi Chiovelli, and Elena Esposito, "Bite and Divide: Malaria and Ethnolinguistic Diversity," 2019. Working paper, University of Bologna.

Cesarini, David, Christopher T. Dawes, James H. Fowler, Magnus Johannesson, Paul Lichtenstein, and Bjorn Wallace, "Heritability of Cooperative Behavior in the Trust Game," Proceedings of the National Academy of Sciences, March 2008, 105 (10), 3721-3726.

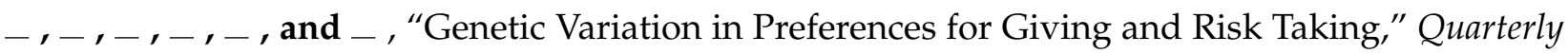
Journal of Economics, May 2009, 124 (2), 809-842.

Chaudhary, Latika, Sriya Iyer, Jared Rubin, and Anand Shrivastava, "Culture and Colonial Legacy: Evidence from Public Goods Games," 2018. Working paper, Naval Postgraduate School.

Chudek, Maciek, Michale Muthukrishna, and Joseph Henrich, "Cultural Evolution," in David M. Buss, ed., Handbook of Evolutionary Psychology, 2nd Edition, New York: Wiley, 2015.

Claessens, Leon P.A.M., Hanneke J.M. Meijer, and Julian P. Hume, "The Morphology of the Thirioux Dodos," Journal of Vertebrate Paleontology, 2015, 35, 29-187.

Clark, Gregory, A Farewell to Alms: A Brief Economic History of the World, Princeton: Princeton University Press, 2007.

Clingingsmith, David, Asim Ijaz Khwaja, and Michael Kremer, "Estimating the Impact of the Hajj: Religion and Tolerance in Islam's Global Gathering," Quarterly Journal of Economics, 2009, 124 (3), 1133-1170. 
Cook, Lisa, "Violence and Economic Growth: Evidence from African American Patents, 18701940," Journal of Economic Growth, 2014, 19 (2), 221-257.

Cornelson, Kirsten, "Media Role Models and Black Educational Attainment: Evidence from the Cosby Show," 2018. Working paper, University of Notre Dame.

Corno, Lucia, Nicole Hildebrandt, and Alessandra Voena, "Age of Marriage, Weather Shocks, and the Direction of Marriage Payments," 2019. Econometrica, forthcoming.

Couttenier, Mattheu, Pauline Grosjean, and Marc Sangnier, "The Wild West is Wild: The Homicide Resource Curse," Journal of the European Economic Association, 2017, 15 (3), 558-585.

Dawkins, Richard, The Blind Watchmaker: Why the Evidence of Evolution Reveals a Universe without Design, New York: W.W. Norton, 1986.

_ , Climbing Mount Improbable, New York: W.W. Norton, 1996.

Dell, Melissa and Pablo Querubin, "The Historical State, Local Collective Action, and Economic Development in Vietnam," Quarterly Journal of Economics, 2018, 133 (2), 701-764.

-, Nathan Lane, and Pablo Querubin, "The Historical State, Local Collective Action, and Economic Development in Vietnam," Econometrica, 2018, 86 (6), 2083-2121.

_ , _, and _, "The Historical State, Local Collective Action, and Economic Development in Vietnam," Econometrica, 2019, 86 (6), 2083-2121.

Della Vigna, Stefano and Ethan Kaplan, "The Fox News Effect: Media Bias and Voting," Quarterly Journal of Economics, 2007, 122, 1187-1234.

Depetris-Chauvin, Emilio, Ruben Durante, and Felipe R. Campante, "Building Nations Through Shared Experiences: Evidence from African Football," American Economic Review, 2020, $110(5), 1572-1602$.

Derex, Maxime, Marie-Pauline Beugin, Bernard Godelle, and Michel Raymond, "Experimental Evidence for the Influence of Group Size on Cultural Complexity," Nature, 2013, 503, 389-391.

Dincecco, Mark and Massimiliano Gaetano Onorato, From Warfare to Wealth, Cambridge: Cambridge University Press, 2017.

Doepke, Matthias and Fabrizio Zilibotti, "Occupational Choice and the Spirit of Capitalism," Quarterly Journal of Economics, 2008, 123 (2), 747-793.

_ and _ "Culture, Entrepreneurship, and Growth," in Philippe Aghion and Steven Durlauf, eds., Handbook of Economic Growth, New York: Elsevier, 2014, pp. 1-48.

_ and _, "Parenting with Style: Altruism and Paternalism in Intergenerational Preference Transmission," Econometrica, 2017, 67 (5), 1331-1337.

Dohmen, Thomas, Armin Falk, David Huffman, and Uwe Sunde, "Patience and Comparative Development," 2018. Working paper, University of Bonn.

Easterly, William, The Elusive Quest for Growth: Economists? Adventures and Misadventures in the Tropics, Cambridge, MA: MIT Press, 2001.

Ehrenfeld, David W. and Archie Carr, "The Role of Vision in the Sea-Finding Orientation of the Green Turtle (Chelonia Myadas)," Animal Behavior, 1967, 15 (1), 25-26. 
Ely, Jeffrey C., "Kludged," American Economic Journal: Microeconomics, 2011, 3, 210-231.

Enke, Benjamin, "Kinship, Cooperation, and the Evolution of Moral Systems," Quarterly Journal of Economics, 2019, 134 (2), 953-1019.

_ , "Moral Values and Voting," forthcoming. Journal of Political Economy.

_ , Ricardo Rodriguez-Padilla, and Florian Zimmerman, "Moral Universalism and the Structure of Ideology," 2019. Working paper, Harvard University.

Fenske, James, "African Polygamy: Past and Present," Journal of Development Economics, 2014, $117,58-73$.

Fernandez, Raquel, "Cultural Change as Learning: The Evolution of Female Labor Force Participation over a Century," American Economic Review, 2013, 103 (1), 472-450.

- Alessandra Fogli, and Claudia Olivetti, "Mothers and Sons: Preference Formation and Female Labor Force Dynamics," Quarterly Journal of Economics, 2004, 119, 1249-1299.

_, Sahar Parsa, and Martina Viarengo, "Coming Out in America: AIDS, Politics, and Cultural Change," 2019. Working paper, New York University.

Figlio, David, Paola Giuliano, Umut Ozek, and Paola Sapienza, "Long-Term Orientation and Educational Performance," American Economic Journal: Economic Policy, 2019, 11 (4), 272-309.

Fouka, Vasiliki, "Backlash: The Unintended Effects of Language Prohibition in U.S. Schools after World War I," Review of Economic Studies, 2020, 87 (1), 204-239.

- and Alain Schlapfer, "Agricultural Returns to Labor and the Origins of Work Ethics," Economic Journal, 2020, 130 (628), 1081-1113.

Francois, Patrick, Thomas Fujiwara, and Tanguy van Ypersele, "Competition Builds Trust," 2010. Working paper, University of British Columbia.

_ , _ , and _ , "The Origins of Human Prosociality: Cultural Group Selection in the Workplace and the Laboratory," Science Advances, 2018, 4 (9), eaat2201.

Friedman, Matt, "The Evolutionary Origin of Flatfish Asymmetry," Nature, 2008, 454, 209-212.

Galef, Bennett G. and Elaine E. Whiskin, "Effects of Environmental Stability and Demonstrator Age on Social Learning of Food Preferences by Young Norway Rats," Animal Behavior, 2004, 68 (4), 897-902.

Galor, Oded and Omer Moav, "Natural Selection and the Origin of Economic Growth," Quarterly Journal of Economics, 2002, 117 (4), 1133-1191.

- and Omer Ozak, "The Agricultural Origins of Time Preference," American Economic Review, 2016, 106 (10), 3064-3103.

- and Stelios Michalopoulos, "Evolution and the Growth Process: Natural Selection of Entrepreneurial Traits," Journal of Economic Theory, 2012, 147 (2), 759-780.

- and Viacheslav Savitskiy, "Climatic Roots of Loss Aversion," 2018. Working paper, Brown University.

Gelfand, Michele, Rule Makers, Rule Breakers, New York: Simon \& Schuster Inc., 2018. 
Gennaioli, Nicola and Hans Joachim Voth, "State Capacity and Military Conflict," Review of Economic Studies, 2015, 82, 1409-1448.

Gershman, Boris, "Witchcraft Beliefs as a Cultural Legacy of the Atlantic Slave Trade: Evidence from Two Continents," 2019. Working paper, American University.

Gigerenzer, Gerd and Daniel G. Goldstein, "Reasoning the Fast and Frugal Way: Models of Bounded Rationality," Psychological Review, 1996, 103 (4), 650-669.

Gintis, Herbert, "A Markov Model of Production, Trade, and Money: Theory and Artificial Life Simulation," Computation E Mathematical Organization Theory, 1997, 3, 19-41.

_ , Game Theory Evolving, New Jersey: Princeton University Press, 2000.

Giuliano, Paola and Antonio Spilimbergo, "Growing Up in a Recession," Review of Economic Studies, 2014, 81 (3), 787-817.

- and Nathan Nunn, "The Transmission of Democracy: From the Village to the Nation State," American Economic Review Papers and Proceedings, 2013, 103 (3), 86-92.

_ and _ , "Understanding Cultural Persistence and Change," forthcoming. Review of Economic Studies.

Gold, Maria Eugenia Leone, Estelle Bourdon, and Mark A. Norell, "The First Endocast of the Extinct Dodo (Raphus Cucullatus) and an Anatomical Comparison Amongst Close Relatives (Aves, Columbiformes)," Zoological Journal of the Linnean Society, 2016, 177, 950-963.

Gorodnichenko, Yuriy and Gerard Roland, "Individualism, Innovation, and Long-Run Growth," Proceedings of the National Academy of Sciences, 2011, 108 (4), 21316-21319.

_ and _, "Culture, Institutions, and Democratization," 2015. Working paper, University of California Berkeley.

_ and _ , "Culture, Institutions, and the Wealth of Nations," Review of Economics and Statistics, 2017, 99 (3), 402-416.

_ , Bohdan Kukharskyy, and Gerard Roland, "Cultural Distance, Firm Boundaries, and Global Sourcing," 2018. Working paper, University of California Berkeley.

Greif, Avner, "Reputation and Coalitions in Medieval Trade: Evidence on the Maghribi Traders," Journal of Economic History, 1989, 49, 857-882.

_ , "Contract Enforceability and Economic Institutions in Early Trade: The Maghribi Traders' Coalition," American Economic Review, 1993, 83 (3), 525-548.

_, "Cultural Beliefs and the Organization of Society: A Historical and Theoretical Reflection on Collectivist and Individualist Societies," Journal of Political Economy, 1994, 102 (5), 912-950.

Grosjean, Pauline, "A History of Violence: The Culture of Honor as a Determinant of Homicide in the U.S. South," Journal of the European Economic Association, 2014, 12 (5), 1285-1316.

_ and Rose Khattar, "It's Raining Men! Hallelujah? The Long-Run Consequences of Male-Biased Sex Ratios," Review of Economic Studies, 2018, 86 (2), 723-754.

Guiso, Luigi, Paola Sapienza, and Luigi Zingales, "Does Culture Affect Economic Outcomes?," Journal of Economic Perspectives, 2006, 20 (2), 23-48. 
_, , , and _ , "Social Capital as Good Culture," Journal of the European Economic Association, 2008, $6(2-3), 295-320$.

_, , , and _, "Cultural Biases in Economic Exchange," Quarterly Journal of Economics, 2009, 124 (3), 1095-1131.

_, , , and _ , "Long-Term Persistence," 2016. Journal of the European Economic Association, forthcoming.

Gustafsson, Anders and Patrik Lindenfors, "Human Size Evolution: No Evolutionary Allometric Relationship Between Male and Female Stature," Journal of Human Evolution, 2004, 47 (4), 253266.

Heldring, Leander, "The Origins of Violence in Rwanda," 2020. Review of Economic Studies, forthcoming.

Heller, Sara B., Anuj K. Shah, Jonathan Guryan, Jens Ludwig, Sendhil Mullainathan, and Harold A. Pollack, "Thinking, Fast and Slow? Some Field Experiments to Reduce Crime and Dropout in Chicago," Quarterly Journal of Economics, 2017, 132 (1), 1-54.

Henrich, Joseph, “Cultural Group Selection, Coevolutionary Processes and Large-Scale Cooperation," Journal of Economic Behavior E Organization, 2004, 53 (1), 3-35.

_ , "Demography and Cultural Evolution: How Adaptive Cultural Processes can Produce Maladaptive Losses - The Tasmanian Case," American Antiquity, 2004, 69 (2), 197-214.

_ , The Secret of Our Success, Princeton: Princeton University Press, 2016.

_, The Weirdest People in the World: How Westerners Became Psychologically Peculiar and Particularly Prosperous 2020.

_, Michal Bauer, Alessandra Cassar, Julie Chytilova, and Benjamin Grant Purzycki, "War Increases Religiosity," Nature Human Behavior, 2019, 3, 129-135.

_, Steven J. Heine, and Ara Norenzayan, "Most People are not WEIRD," Nature, 2010, 466 (29).

_ , _ , and _ , "The Weirdest People in the World," Behavioral and Brain Sciences, 2010, 33 (2/3), $1-75$.

Hume, Julian P., "The History of the Dodo Raphus Cucullatus and the Penguin of Mauritius," Historical Biology, 2006, 18 (2), 69-93.

Jakiela, Pamela and Owen Ozier, "The Impact of Violence on Individual Risk Preferences: Evidence from a Natural Experiment," Review of Economics and Statistics, 2019, 101 (3), 547-559.

Jayachandran, Seema and Rohini Pande, "Why Are Indian Children So Short? The Role of Birth Order and Son Preference," American Economic Review, 2017, 107 (9), 2600-2629.

Jensen, Robert and Emily Oster, "The Power of TV: Cable Television and Women's Status in India," Quarterly Journal of Economics, 2009, 124 (3), 1057-1094.

Kahneman, Daniel, Thinking, Fast and Slow, London: Macmillan, 2011.

Kamarck, Andrew M., Culture Under Cross-Examination: International Justice and the Special Court for Sierra Leone, New York: Cambridge University Press, 2009. 
Karaja, Elira and Jared Rubin, "The Cultural Transmission of Trust Norms: Evidence from a Lab in the Field on a Natural Experiment," 2017. Working paper, Chapman University.

Katz, S.H., M.L. Hediger, and L.A. Valleroy, "Traditional Maize Processing Techniques in the New World," Science, 1974, 184 (4138), 765-773.

Kawai, Keiichi, Ruitian Lang, and Hongyi Li, "Political Kludges," American Economic Journal: Microeconomics, 2018, 10 (4), 131-158.

Kine, Michelle A. and Robert Boyd, "Population Size Predicts Technological Complexity in Oceania," Proceedings of the Royal Society B, 2010.

Kobayashi, Yutaka and Kenichi Aoki, "Innovativeness, Population Size, and Cumulative Cultural Evolution," Theoretical Population Biology, 2012, 82, 38-47.

Kosse, Fabian, Thomas Deckers, Hannah Schildberg-Horisch, and Armin Falk, "The Formation of Prosociality: Causal Evidence on the Role of the Social Environment," 2019. Journal of Political Economy, forthcoming.

Kremer, Michael, "Population Growth and Technological Change: One Million B.C. to 1990," Quarterly Journal of Economics, 1993, 108 (3), 681-716.

La Ferrara, Eliana, Alberto Chong, and Suzanne Duryea, "Soap Operas and Fertility: Evidence from Brazil," American Economic Journal: Applied Economics, 2012, 4 (4), 1-31.

Long, Jason and Joseph Ferrie, "Occupational Mobility in Great Britain and the United States since 1850," American Economic Review, 2013, 103 (4), 1109-1137.

Lowes, Sara, "Matrilineal Kinship and Spousal Cooperation: Evidence from the Matrilineal Belt," 2018. Working paper, Stanford University.

- and Eduardo Montero, "Mistrust in Medicine: The Legacy of Colonial Medicine Campaigns in Central Africa," 2017. Working paper, Bocconi University.

_ and _ , "Concessions, Violence and Indirect Rule: Evidence from the Congo Free State," 2019. Working paper, Stanford University.

_ , Nathan Nunn, James A. Robinson, and Jonathan Weigel, "The Evolution of Culture and Institutions: Evidence from the Kuba Kingdom," Econometrica, 2017, 85 (4), 1065-1091.

Madestam, Andreas and David Yanagizawa-Drott, "Shaping the Nation: The Effect of the Fourth of July on Political Preferences and Behavior in the United States," 2011. Working paper, Harvard University.

_, Daniel Shoag, Stan Veuger, and David Yanagizawa-Drott, "Do Political Protests Matter? Evidence from the Tea Party Movement," Quarterly Journal of Economics, 2013, 128 (4), 1633-1685.

Mankiw, N. Gregory, David Romer, and David N. Weil, "A Contribution to the Empirics of Economic Growth," Quarterly Journal of Economics, 1992, 107, 407-437.

McElreath, Richard, Mark Lubell, Peter J. Richerson, Timothy M. Waring, William Baum, Edward Edstein, Charles Efferson, and Brian Paciotti, "Applying Evolutionary Models to the Laboratory Study of Social Learning," Evolution and Human Behavior, 2005, 26, 483-508.

Mesoudi, Alex, "Cultural Evolution: A Review of Theory, Findings and Controversies," Evolutionary Biology, 2016, 43, 481-497. 
Michalopoulos, Stelios, "The Origins of Ethnolinguistic Diversity," American Economic Review, 2012, 102 (4), 1508-1539.

- , Alireza Naghavi, and Giovanni Prarolo, "Trade and Geography in the Spread of Islam," Economic Journal, 2018, 128 (616), 3210-3241.

Mitchener, Kris James and Ian W. McLean, "The Productivity of U.S. States Since 1880," Journal of Economic Growth, 2003, 8, 73-114.

Mitrunen, Matti, "Can you Make an American? Compulsory Patriotism and Assimilation of Immigrants," 2019. Working paper, University of Chicago.

Mokyr, Joel, A Culture of Growth: The Origins of the Modern Economy, Princeton: Princeton University press, 2018.

Moscona, Jacob, Nathan Nunn, and James A. Robinson, "Keeping It in the Family: Lineage Organization and the Scope of Trust in Sub-Saharan Africa," American Economic Review Papers and Proceedings, 2017, 107 (5), 565-571.

$\ldots, \ldots$, and $\_,$"Kinship and Conflict: Evidence from Segmentary Lineage Societies in SubSaharan Africa," 2019. Econometrica, forthcoming.

Muthukrishna, Michael, Adrian V. Bell, Joseph Henrich, Cameron M. Curtin, Alexander Gedranovich, Jason McInerney, and Braden Thue, "Beyond Western, Educated, Industrial, Rich, and Democratic (WEIRD) Psychology: Measuring and Mapping Scales of Cultural and Psychological Distance," Psychological Science, 2020, 31 (6), 678-701.

- and Edward Slingerland, "Psychology as a Historical Science," Annual Review of Psychology, forthcoming.

- and Joseph Henrich, "Innovation and the Collective Brain," Philosophical Transactions B, 2016, $371,20150192$.

_, Ben W. Shulman, Vlad Vasilescu, and Joseph Henrich, "Sociality Influences Cultural Complexity," Proceedings of the Royal Society B, 2014, 281, 20132511.

Nguyen, Kieu-Trang, "Trust and Innovation within the Firm: Evidence from Matched CEO-Firm Data," 2018. Working paper, London School of Economics.

Norman, J.R., A Systematic Monograph of the Flatfishes (Heterosomata). Vol. 1. Psettotidae, Bothidae, Pleuronectidae, London: The Trustees of the British Museum, 1934.

Nunn, Nathan, "Historical Legacies: A Model Linking Africa's Past to its Current Underdevelopment," Journal of Development Economics, 2007, 83 (1), 157-175.

_, "Slavery, Inequality, and Economic Development in the Americas: An Examination of the Engerman-Sokoloff Hypothesis," in Elhanan Helpman, ed., Institutions and Economic Performance, Cambridge, MA: Harvard University Press, 2008, pp. 148-180.

_ , "Religious Conversion in Colonial Africa," American Economic Review Papers and Proceedings, 2010, 100 (2), 147-152.

_ , "On the Causes and Consequences of Cross-Cultural Differences: An Economic Perspective, Volume 8," in Michele J. Gelfand, Chi-Yue Chiu, and Ying-Yi Hong, eds., Advances in Culture and Psychology, Oxford: Oxford University Press, 2021, p. forthcoming. 
- and Leonard Wantchekon, "The Slave Trade and the Origins of Mistrust in Africa," American Economic Review, 2011, 101 (7), 3221-3252.

- and Raul Sanchez de la Sierra, "Why Being Wrong Can Be Right: Magical Warfare Technologies and the Persistence of False Beliefs," American Economic Review Papers and Proceedings, 2017, 107 (5), 582-587.

_, Nancy Qian, and Jaya Wen, "Distrust and Political Turnover," 2019. Working paper, Harvard University.

Okoye, Dozie, “Things Fall Apart? Missions, Institutions, and Interpersonal Trust?," 2017. Working paper, Dalhousie University.

Olken, Benjamin, "Do Television and Radio Destroy Social Capital?," American Economic Journal: Applied Economics, 2009, 1 (4), 1-33.

Platteau, Jean-Philippe, Microeconomics: Behavior, Institutions, and Evolution, Singapore: Harwood Academic Publishers, 2000.

Qian, Nancy, "Missing Women and the Price of Tea in China: The Effects of Sex-Specific Income on Sex Imbalance," Quarterly Journal of Economics, 2008, 123 (3), 1251-1285.

Ramos-Toro, Diego, "Social Exclusion and Social Preferences: Evidence from Colombia's Leper Colony," 2019. Working paper, Brown University.

Rao, Gautam, "Familiarity Does Not Breed Contempt: Diversity, Discrimination, and Generosity in Delhi Schools," American Economic Review, 2019, 109 (3), 774-809.

Richerson, Peter, Ryan Baldini, Adrian V. Bell, Kathryn Demps, Karl Frost, Vicken Hillis, Sarah Mathew, Emily K. Newton, Nicole Naar, Lesley Newson, Cody Ross, Paul E. Smaldino, Timothy M. Waring, and Matthew Zefferman, "Cultural Group Selection Plays and Essential Role in Explaining Human Cooperation: A Sketch of the Evidence," Behavioral and Brain Sciences, 2016, 39, e30.

Riley, Emma, "Increasing Students' Aspirations: The Impact of Queen of Katwe on Students' Educational Attainment," 2017. CSAE Working Paper WPS/207-13.

Rogers, Alan R., "Does Biology Constrain Culture?," American Anthropologist, 1988, 90 (4), 819831.

Rubin, Jared, "Printing and Protestants: An Empirical Test of the Role of Printing in the Reformation," Review of Economics and Statistics, 2014, 96 (2), 270-286.

Ruff, Christopher, "Variation in Human Body Size and Shape," Annual Review of Anthropology, 2002, 31, 211-232.

Rustagi, Devesh, "Historical Self-Governance and Norms of Cooperation," 2019. Working paper, Brown University.

Sacerdote, Bruce, "How Large Are the Effects from Changes in Family Environment? A Study of Korean American Adoptees," Quarterly Journal of Economics, 2007, 122 (1), 119-157.

Salmon, Michael, Melissa Garro Tolbert, Danielle Pender Painter, Matthew Goff, and Raymond Reiners, "Behavior of Loggerhead Sea Turtles on an Urban Beach. II. Hatchling Orientation," Journal of Herpetology, 1995, 29 (4), 568-576. 
Schulz, Jonathan, "The Churches' Bans on Consanguineous Marriages, Kin Networks and Democracy," 2017. Working paper, Yale University.

- , Duman Bahrami-Rad, Jonathan Beauchamp, and Joseph Henrich, "The Church, Intensive Kinship, and Global Psychological Variation," Science, 2019, 366 (6466), eaau5141.

Sequeira, Sandra, Nathan Nunn, and Nancy Qian, "Immigrants and the Making of America," Review of Economic Studies, 2020, 87 (1), 382-419.

Sinclair-Thomas, Brent and Sam Challis, "The 'Bullets to Water' Belief Complex: A PanSouthern African Cognate Epistemology for Protective Medicines and the Control fo Projectiles," Journal of Conflict Archaeology, 2017, 12 (3), 192-208.

Speck, Frank G., Naskapi: The Savage Hunters of the Labrador Peninsula, Norman: University of Oklahoma Press, 1935.

Spolaore, Enrico and Romain Wacziarg, "The Diffusion of Development," Quarterly Journal of Economics, 2009, 124 (2), 469-529.

Tabellini, Guido, "The Scope of Cooperation: Values and Incentives," Quarterly Journal of Economics, 2008, 123 (3), 905-950.

Talhelm, Thomas, X. Zhang, S. Oishi, C. Shimin, D. Duan, X. Lan, and X. Kitayama, "LargeScale Psychological Differences Within China Explained by Rice Versus Wheat Agriculture," Science, 2014, 344 (6184), 603-608.

Teso, Edoardo, "The Long-Term Effects of Demographic Shocks on the Evolution of Gender Roles: Evidence from the Trans-Atlantic Slave Trade," Journal of the European Economic Association, 2019, 17 (2), 497-534.

Tilly, Charles, Coercion, Capital and European States, A.D. 990-1990, Cambridge: Blackwell Publishers, 1990.

Todd, Gerd Gigerenzer Peter M. and The ABC Research Group, Simple Heuristics that Make Us Smart, Oxford: Oxford University Press, 1999.

Toelch, Ulf, Marjolijn J. van Delft, Matthew J. Bruce, Rogier Donders, Marius T.H. Meeus, and Simon M. Reader, "Decreased Environmental Variability Induces a Bias for Social Information Use in Humans," Evolution and Human Behavior, 2009, 30 (1), 32-40.

Trivers, Robert L., "Parental Investment and Sexual Selection," in B. Campbell, ed., Sexual Selection and Descent of Man, 1871-1971, Chicago: Aldine, 1972, pp. 136-179.

Tur-Prats, Ana and Felipe Valencia Caicedo, "The Long Shadow of the Spanish Civil War," 2020. Working paper, University of British Columbia.

Turchin, Peter, Historical Dynamics: Why States Rise and Fall, Princeton: Princeton University Press, 2003.

_ , War and Peace and War: The Life Cycles of Imperial Nations, New York: Pi Press, 2006.

_ , How 10,000 Years of War Made Humans the Greatest Cooperators on Earth, Chaplin, Connecticut: Beresta Books, 2016.

_ , Thomas E. Currie, Edward A. L. Turner, and Sergey Gavrilets, "War, space, and the evolution of Old World complex societies," Proceedings of the National Academy of Sciences, 2013, 110, 1638416389 . 
Vaesen, Krist, “Cumulative Cultural Evolution and Demography," PLoS One, 2012, 7 (7), e40989.

Vehrencamp, Sandra L., "A Model for the Evolution of Despotic Versus Egalitarian Societies," Animal Behavior, 1983, 31, 667-682.

Xue, Melanie Meng, "High-Value Work and the Rise of Women: The Cotton Revolution and Gender Equality in China," 2018. Working paper, Northwestern University. 